\title{
Infrared to x-ray spectral energy distributions of high redshift quasars
}

\section{Citation}

Bechtold, Jill, Martin Elvis, Fabrizio Fiore, Olga Kuhn, Roc M. Cutri, Jonathan C. McDowell, Marcia Rieke, Aneta Siemiginowska, and Belinda J. Wilkes. 1994. "Infrared to x-Ray Spectral Energy Distributions of High Redshift Quasars." The Astronomical Journal 108 (August): 374. doi:10.1086/117076.

\section{Published Version}

doi:10.1086/117076

\section{Permanent link}

http://nrs.harvard.edu/urn-3:HUL.InstRepos:30212176

\section{Terms of Use}

This article was downloaded from Harvard University's DASH repository, and is made available under the terms and conditions applicable to Other Posted Material, as set forth at http:// nrs.harvard.edu/urn-3:HUL.InstRepos:dash.current.terms-of-use\#LAA

\section{Share Your Story}

The Harvard community has made this article openly available.

Please share how this access benefits you. Submit a story.

\section{Accessibility}




\title{
INFRARED TO X-RAY SPECTRAL ENERGY DISTRIBUTIONS OF HIGH REDSHIFT QUASARS
}

\author{
JILL BECHTOLD \\ Steward University, University of Arizona, Tucson, Arizona 85721 \\ Electronic mail: jbechtold@as.arizona.edu \\ MARTin Elvis, Fabrizio FIORE, AND Olga KuHN \\ Harvard-Smithsonian Center for Astrophysics, 60 Garden Street, Cambridge, Massachusetts 02138 \\ Electronic mail: elvis@cfa.harvard.edu \\ ROC M. CUTRI \\ Steward Observatory, University of Arizona, Tucson, Arizona 85721 \\ Electronic mail: rcutri@as.arizona.edu \\ JONATHAN C. MCDOWELL \\ Harvard-Smithsonian Center for Astrophysics, 60 Garden Street, Cambridge, Massachusetts 02138 \\ Electronic mail: mcdowell@as.arizona.edu \\ MARCIA RIEKE \\ Steward Observatory, University of Arizona, Tucson, Arizona 85721 \\ Electronic mail: mrieke@as.arizona.edu \\ ANETA SiemiginowsKa AND Belinda J. WiLKes \\ Harvard-Smithsonian Center for Astrophysics, 60 Garden Street, Cambridge, Massachusetts 02138 \\ Received 1994 February 14; revised 1994 April 18
}

\begin{abstract}
We have observed 14 quasars with $z>2.8$ with the ROSAT-PSPC, and detected 12 of them, including the $z=4.11$ quasar $0000-263$. We present the first x-ray spectrum of a radio quiet quasar with $z>3,1946+768$. Its $\mathrm{x}$-ray spectrum is consistent with a power law with spectral index $\alpha_{E}=1.8_{-1.4}^{+2.1}$ and no evidence for absorption in excess of the galactic column $\left[\alpha_{E}=1.00_{-0.32}^{+0.28}\right.$ assuming $\left.N_{\mathrm{H}}=N_{\mathrm{H}}(\mathrm{Gal})\right]$. A PSPC hardness ratio is used to constrain the $\mathrm{x}$-ray spectral properties of the quasars for which there were less than 100 photons detected. For the radio quiet quasars, $\left\langle\alpha_{E}\right\rangle \approx 1.2$, if one assumes that there is no absorption in excess of the galactic column. We combine the x-ray data with new ground based optical and near-IR spectrophotometry obtained at the Steward $2.3 \mathrm{~m}$ and Multiple Mirror Telescope, and data from the literature. The spectral energy distributions are compared to those of low redshift objects. For the radio quiet quasars with $z>2.5$, the mean $\left\langle\alpha_{\text {ox }}\right\rangle \sim 1.8$. This is larger than the mean for quasars with $z<2.5$, but consistent with the expected value for quasars with the high optical luminosities of the objects in this sample. For the radio-loud quasars, $\left\langle\alpha_{\mathrm{ox}}\right\rangle \sim 1.4$, independent of redshift. This is smaller than the expected value for the optically luminous, high redshift objects in this sample, if they are mostly $\mathrm{GHz}$ peaked radio sources and hence comparable to steep-spectrum, compact radio sources at lower redshift. Finally, we compare the spectral energy distributions of two representative objects to the predicted spectrum of a thin accretion disk in the Kerr geometry, and discuss the uncertainties in deriving black hole masses and mass accretion rates.
\end{abstract}

\section{INTRODUCTION}

In recent years, the spectral energy distributions of many low-redshift quasars have been observed from the soft $\mathrm{x}$-rays to far-IR and radio wavelengths (Elvis et al. 1986; Neugebauer et al. 1987; Sanders et al. 1989; Elvis et al. 1994b, and references therein). While these multiwavelength studies may have raised more new questions than they have answered, they have surely been invaluable stimulis for discussion and theoretical work. In particular, the x-ray fluxes and spectra have been key to exploring such issues as the nature of emission from the accretion disk or the "big blue bump" (Sun \& Malkan 1989), the origin and energy budget of the
Fe II emission and other broad emission lines (Wilkes et al. 1987; Netzer 1990, and references therein), the possible existence of nonthermal components to the IR emission (Green et al. 1992), the physical conditions in the radio emitting plasma (Worrall et al. 1987), and the contribution of quasars to the cosmic x-ray background (Maccacaro et al. 1991, and references therein).

To date most studies have been limited to quasars with $z<0.5$. Given the evolution in the ensemble properties of quasars, one expects to see changes in the continuum properties of individual objects at higher redshifts. The optical quasar luminosity function has a strong cutoff in the numbers of quasars at $z>3$, presumably resulting from the formation 


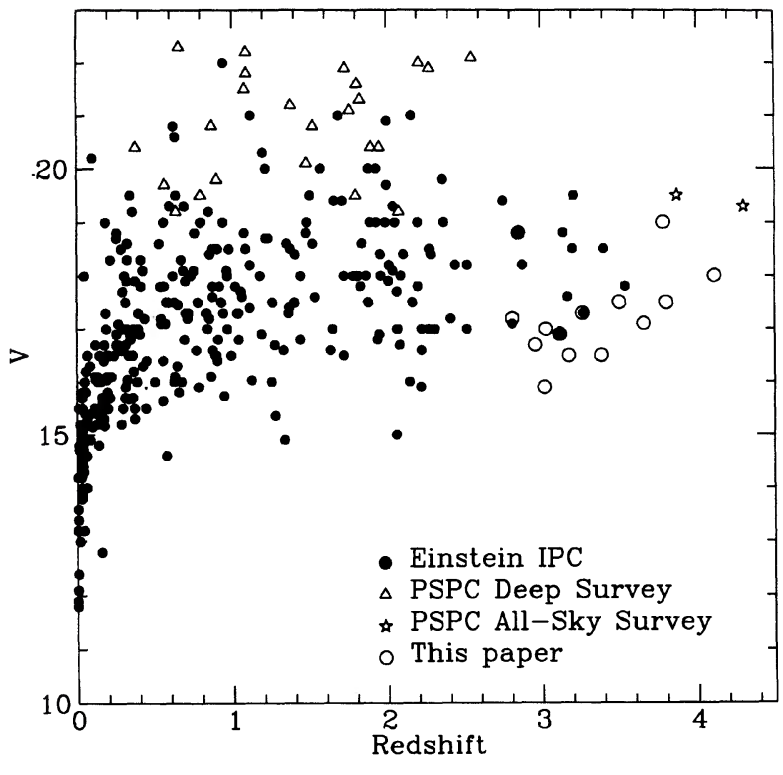

FIG. 1. Apparent $V$ magnitude vs redshift for quasars detected by the Einstein Observatory IPC (filled circles), Shanks et al. (1991) deep ROSAT survey (open triangles), and this work (open circles). Quasars from the PSPC all-sky survey are plotted with the star symbol (see text).

and subsequent evolution of the central engine and accretion disk (Boyle 1993, and references therein). Results for the $\mathrm{x}$-ray luminosity function imply evolution of $\mathrm{x}$-ray properties at somewhat smaller redshifts, $z \approx 1-2$ (Della Ceca \& Maccacaro 1991; Shanks et al. 1991; Hasinger et al. 1991; Boyle et al. 1993). The fraction of quasars that are radio-loud decreases with increasing redshift (Schneider et al. 1992b; Visnovsky et al. 1992). Our understanding of the nature of these changes may be advanced through detailed observations of the spectral energy distributions of individual high redshift objects.

In this paper, we present ROSAT PSPC observations and new near-IR and optical observations of 14 bright, high redshift quasars. These objects all have $z>2.8$, and are among the most luminous objects in the sky. Almost all have been discovered since the demise of the Einstein Observatory, and so had not been studied previously in $\mathrm{x}$-rays. While this sample is admittedly heterogeneous in terms of the way the objects were selected, we do not expect a comparable sample of high redshift quasars to be found in more statistically complete x-ray surveys. The ROSAT all-sky survey is too shallow to detect many $z>3$ quasars, although recently two have been detected (Fink \& Briel 1993; Henry et al. 1994). The deep pointed PSPC surveys (e.g., Shanks et al. 1991) cover too little of the sky to include many of these rare objects. Pointed observations of known objects with relatively long exposure times were necessary to detect even the brightest high redshift examples. Figure 1 shows the Hubble diagram for all quasars detected with the Einstein Observatory (filled circles), the quasars in the deep ROSAT survey of Shanks et al. (1991) (triangles), the two high-redshift quasars found in the ROSAT all-sky survey (Fink \& Briel 1993;
TABLE 1. Log of ROSAT PSPC observations.

\begin{tabular}{|c|c|c|c|c|c|c|c|}
\hline Quasar & $z_{\epsilon m}{ }^{a}$ & $\mathrm{~V}^{a}$ & $\mathrm{RL}^{b}$ & ID & Date & $\begin{array}{l}\text { SASS } \\
\text { Version }\end{array}$ & $\begin{array}{r}\text { Exposure } \\
\text { (s) }\end{array}$ \\
\hline $0000-263^{c}$ & 4.111 & 18 & $<-0.37$ & гр700078 & 30 Nov 91 & $5 \_6$ & 4298 \\
\hline $0014+813^{c}$ & 3.387 & 16.5 & $2.8 \pi$ & rp 700083 & 16-17 Mar 91 & $5 \_3 \_2$ & 5951 \\
\hline $0055-264$ & 3.66 & 17.1 & -0.04 & rp 700081 & 28 Dec 91 & $5 \_6$ & 3862 \\
\hline $0130-403$ & 3.03 & $1 \overline{7} .0$ & - & гр701031 & 29-31 Dec 92 & $6 \_2$ & 6214 \\
\hline $0207-398$ & 2.813 & 17.2 & - & rpī0002T & 13 Nov 92 & $6 \_2$ & 25030 \\
\hline $0420-388^{c}$ & 3.12 & 16.9 & 2.47 & rр700026 & 20-22 Feb 91 & $5 \_3 \_2$ & 11896 \\
\hline \multirow[t]{2}{*}{$0438-436^{c}$} & 2.852 & 18.8 & 4.49 & rp 700028 & 19-21 Feb 91 & $5 \_3$ & 10725 \\
\hline & & & & гр700867 & 19-20 Sep 92 & $6 \_2$ & 10506 \\
\hline $0636+680^{c}$ & 3.178 & 16.5 & 2.10 & rр 700084 & $16 \operatorname{Mar} 91$ & $5 \_2$ & 5342 \\
\hline $1107+481$ & 2.96 & 16.7 & -0.38 & rp700297 & 15-16 May 92 & 5.7 & 3060 \\
\hline $1159+123$ & 3.502 & 17.5 & $<-0.20$ & rp7010300 & 8 Dec 92 & 62 & 1812 \\
\hline $1208+101$ & 3.803 & 17.5 & $<-0.13$ & $\operatorname{rp} 700079$ & $\begin{array}{c}3 \text { June } 92 \\
16-17 \text { Dec } 91\end{array}$ & 5.7 & $\begin{array}{l}2829 \\
2999\end{array}$ \\
\hline $1946+768^{c}$ & 3.02 & 15.9 & -0.56 & rpī01410 & 24-25 June 93 & $6 \_4$ & 13876 \\
\hline $2000-330^{c}$ & 3.783 & 19.0 & 4.02 & rр700080 & 31 Mar 91 & 5_3_2 & 3740 \\
\hline \multirow[t]{2}{*}{$2126-158^{c}$} & 3.266 & 17.3 & 3.06 & rp700025 & 9 May 91 & 5_3_2 & 3424 \\
\hline & & & & rp700868 & 12-13 Nov 92 & $6 \_.$ & 3968 \\
\hline
\end{tabular}

Notes to TABLE 1

${ }^{\text {a }}$ Hewitt $\&$ Burbidge (1993), except $z_{\mathrm{em}}$ for $1107+481$ is from data presented here.

${ }^{\mathrm{b}} R L$ is radio loudness; see Eq. (1). We call quasars with $R L>1$ radio loud, and those with $R L \leqslant 1$ radio quiet.

${ }^{c}$ Results of spectral fits are given in Table 6 or Paper I.

Henry et al. 1994; open stars), and the objects in our sample (open circles).

Finally, the bright apparent magnitudes and high redshifts of these objects have generated great interest in them (three of them held the record for highest known redshift quasar when they were discovered), so there is a rich literature of related observations at many wavelengths. All of these quasars have extensively studied optical absorption-line spectra, which are useful for measuring the continuum in the gaps between the lines in the Ly $\alpha$ forest, and for identifying intervening galaxies such as damped Ly $\alpha$ absorbers which may significantly redden the intrinsic quasar continuum. In addition, a few are bright enough to have been detected with IRAS, and in the submillimeter (see Sec. 5). A discussion of the x-ray spectra of six of these objects can be found in Elvis et al. (1994a, hereafter referred to as Paper I), and new mid-IR measurements and radio fluxes are presented in Kuhn et al. (1994) and Kuhn (1994). Here, in addition to the x-ray results, we present near-IR spectra of these objects obtained with the Germanium Spectrometer at the MMT. The infrared data are valuable, since the $1-2 \mu \mathrm{m}$ region corresponds to rest wavelengths of 2500 to $5000 \AA$, the spectral region of the "big blue bump" and the well studied optical emission lines.

\section{X-RAY DATA}

\subsection{ROSAT PSPC Observations}

In Table 1, we list the data obtained with ROSAT (Trumper 1983) and the Position Sensitive Proportional Counter (PSPC, Pfefferman et al. 1987) between March 1991 and June 1993. The PSPC data for $0014+813,0420$ $-388,0438-436,0636+580,2000-330$, and 2126-158, were discussed previously in Paper I. For reference, we also list the radio loudness for each object, $R L$, defined as

$$
R L=\log [S(5 \mathrm{GHz}) / S(\lambda 1450)],
$$


TABLE 2. PSPC results: observed counts and hardness ratios.

\begin{tabular}{|c|c|c|c|c|c|c|c|}
\hline Quasar & $z_{e m}$ & $\begin{array}{r}\text { Exposure } \\
(\mathrm{s})\end{array}$ & $\underset{,}{\text { Radius }}$ & $\begin{array}{l}\text { Total } \\
\text { PI 11-245 }\end{array}$ & $\begin{array}{c}\text { Counts } \\
\text { Soft } \\
11-40\end{array}$ & $\begin{array}{c}\text { Hard } \\
41-245\end{array}$ & $\begin{array}{c}\text { Ratio } \\
\text { (Hard-Soft)/Total }\end{array}$ \\
\hline $0000-263$ & 4.11 & 4298 & 1.5 & $25.8 \pm 7.9$ & $13.8 \pm 6.3$ & $12.1 \pm 4.8$ & $-0.065 \pm 0.306$ \\
\hline $0000-263$ & 4.11 & 37420 & 2.0 & $161.5 \pm 29.5$ & $86.0 \pm 24.0$ & $75.5 \pm 1 \overline{7} .1$ & $-0.065 \pm 0.183$ \\
\hline $0014+813$ & 3.38 & 5951 & 2.0 & $398.9 \pm 22.1$ & $7.5 \pm 7.2$ & $391.4 \pm 20.9$ & $0.962 \pm 0.077$ \\
\hline 0130-403 & 3.03 & 6214 & 1.5 & $50.1 \pm 10.9$ & $29.3 \pm 9.2$ & $20.8 \pm 15.9$ & $-0.170 \pm 0.372$ \\
\hline $020 \bar{\tau}-398^{a}$ & 2.81 & 25030 & 1.0 & $6 \pi .6 \pm 14.2$ & $29.2 \pm 12.3$ & $37.9 \pm 8.1$ & $0.129 \pm 0.221$ \\
\hline $020 T-398^{b}$ & 2.81 & 8246 & 1.0 & $35.4 \pm 8.6$ & $17.4 \pm \tau .5$ & $18.4 \pm 5.1$ & $0.028 \pm 0.253$ \\
\hline $020 \mathrm{~T}-398^{c}$ & 2.81 & 17611 & 1.0 & $32.2 \pm 11.2$ & $11 . \tau \pm 9.8$ & $19.5 \pm 6.3$ & $0.250 \pm 0.385$ \\
\hline $0420-388$ & 3.12 & $896^{d}$ & 2.0 & $36 \pi .6 \pm 25.2$ & $109.1 \pm 16.7$ & $258.5 \pm 18.9$ & $0.406 \pm 0.074$ \\
\hline & 2.85 & $21231^{d}$ & 2.0 & $1163.0 \pm 43.8$ & $76.0 \pm 25.0$ & $1097.9 \pm 35.4$ & $0.871 \pm 0.049$ \\
\hline $0438-436^{e}$ & 2.85 & $10725^{d}$ & 2.0 & $636.7 \pm 34.8$ & $31.5 \pm 20.9$ & $618.6 \pm 27.3$ & $0.903 \pm 0.071$ \\
\hline $0438-436^{f}$ & 2.85 & 10506 & 2.0 & $523.8 \pm 26.5$ & $44.5 \pm 13.8$ & $479.3 \pm 22.6$ & 0.066 \\
\hline $0636+680$ & 3.17 & 5342 & 2.0 & $68.8 \pm 12.5$ & $-4.8 \pm 6.5$ & $73.6 \pm 10.7$ & $1.140 \pm 0.276$ \\
\hline $110 \tau+481$ & 2.96 & 3060 & 1.5 & $29.2 \pm 7.5$ & $13.6 \pm 5.9$ & $15.6 \pm 4.6$ & $0.0685 \pm 0.257$ \\
\hline $1159+123$ & 3.53 & & 1.5 & $<12.7$ & & & \\
\hline $1208+101$ & 3.82 & & 1.5 & $29.3 \pm 9.8$ & $18.3 \pm 8.4$ & & $-0.249 \pm$ \\
\hline $1946+i 68$ & 3.02 & 13876 & 1.0 & $94.7 \pm 10.9$ & & & $0.935 \pm 0.157$ \\
\hline & 3.78 & $3 \pi 40$ & 2.0 & $63.1 \pm 12.4$ & & & $0.721 \pm 0.242$ \\
\hline $2126-158^{a}$ & 3.27 & 7392 & 2.0 & $1259.5 \pm 37.2$ & $23.0 \pm 10.0$ & $1236.5 \pm$ & $0.963 \pm 0.041$ \\
\hline & 3.27 & 3424 & 2.0 & $562.1 \pm 24.3$ & & $569.7 \pm 25.3$ & $0.974 \pm 0.063$ \\
\hline $2126-158^{h}$ & 3.27 & 3968 & 2.0 & $39.8 \pm 27.3$ & $15.5 \pm 7.1$ & $674.3 \pm 26.4$ & $0.955 \pm 0.055$ \\
\hline
\end{tabular}

Notes to TABLE 2

${ }^{a}$ All data summed together. ${ }^{b}$ Data of $11 / 91$ only. ${ }^{c}$ Data of $7 / 92$ only. ${ }^{d}$ Wobble off for part of the observation. ${ }^{\mathrm{e}}$ Data of $2 / 91$ only. ${ }^{\mathrm{f}}$ Data of $9 / 92$ only. ${ }^{\mathrm{g}}$ Data of $5 / 91$ only. hata of $11 / 92$ only.

where $S(5 \mathrm{GHz})$ is the radio flux at an observed frequency of $5 \mathrm{GHz}$, and $S(\lambda 1450)$ is the flux at $\lambda 1450 \AA$ in the rest frame. We call quasars with $R L>1$ radio-loud, and those with $R L \leqslant 1$, radio-quiet. References for the radio fluxes are given in Sec. 5.

Tables 2 and 3 summarize the observed $x$-ray properties of the program objects. To measure the x-ray fluxes, the counts within $1^{\prime}, 1.5^{\prime}$, and $2^{\prime}$ of the optical source position were summed using PROS routines in IRAF. The background was estimated by summing the counts within an annulus (with inner and outer radii of $3^{\prime}$ and $5^{\prime}$, respectively), with the area around strong sources removed. Table 2 lists the total counts in PI channels 11-245 (corresponding to observed energies of approximately 0.1 to $2.4 \mathrm{keV}$ ) for the radius $\left(1^{\prime}, 1.5^{\prime}\right.$, or $\left.2^{\prime}\right)$ which gave the highest signal-tonoise. Because of the dependence of the PSPC point spread

TABLE 3. PSPC fluxes.

\begin{tabular}{lccccccc}
\hline \hline Quasar & \multirow{2}{*}{$\mathrm{z}_{\text {em }}$} & \multirow{2}{*}{$\mathrm{N}_{\text {Gal }}{ }^{a}$} & \multicolumn{5}{c}{$\mathrm{f}_{x}$ for Spectral Model } \\
& & & $\# 1$ & $\# 2$ & $\# 3$ & $\# 4$ & $\begin{array}{c}\text { \% } \\
\text { Error }^{c}\end{array}$ \\
\hline $0000-263^{d}$ & 4.11 & 1.60 & 2.23 & 2.03 & 1.70 & 2.92 & 30 \\
$0000-263^{e}$ & 4.11 & 1.60 & 1.60 & 1.46 & 1.22 & 2.10 & 18 \\
$0014+813$ & 3.38 & 14.4 & 48.8 & 49.7 & 50.6 & 53.1 & 6 \\
$0130-403$ & 3.03 & 2.30 & 3.37 & 3.14 & 2.74 & 4.57 & 22 \\
$0207-398$ & 2.81 & 1.53 & 1.01 & 0.92 & 0.76 & 1.56 & 21 \\
$0420-388$ & 3.12 & 1.91 & 12.2 & 11.2 & 9.56 & 17.1 & 7 \\
$0438+436$ & 2.35 & 1.50 & 18.2 & 16.4 & 13.7 & 27.9 & 54 \\
$0636+680$ & 3.17 & 5.70 & 7.13 & 7.04 & 6.81 & 8.17 & 18 \\
$1107+481$ & 2.96 & 1.40 & 3.40 & 3.06 & 2.53 & 5.22 & 26 \\
$1159+123$ & 3.53 & 2.10 & $<3.51$ & $<3.25$ & $<2.81$ & $<4.62$ & \\
$1208+101$ & 3.82 & 1.70 & 1.90 & 1.73 & 1.46 & 2.54 & 33 \\
$1946+768$ & 3.02 & 7.50 & 4.08 & 4.07 & 4.03 & 4.60 & 10 \\
$2000-330$ & 3.78 & 7.50 & 10.1 & 10.1 & 9.95 & 11.0 & 20 \\
$2126-158$ & 3.27 & 4.85 & 90.0 & 88.2 & 84.0 & 104.4 & 3 \\
\hline
\end{tabular}

Notes to TABLE 3

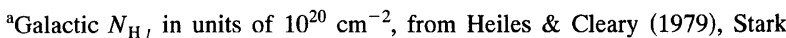
et.al. (1989), and Elvis et al. (1989). ${ }^{\mathrm{b}} f_{\mathrm{x}}=$ flux at $1 \mathrm{keV}$ in $10^{-5}$ photons $\mathrm{cm}^{-2} \mathrm{~s}^{-1} \mathrm{keV}^{-1}$. Note $f(\mathrm{mJy})=f_{\mathrm{x}} \times 662 \times 10^{-5}$. Spectral Model \#1 has $\alpha_{E}=0.5$ and $N_{\mathrm{H}}=N_{\mathrm{Gal}}$. Model \#2 has $\alpha_{E}=0.7$ and $N_{\mathrm{H}}=N_{\mathrm{Gal}}$. Model \#3 has $\alpha_{E}=1.0$ and $N_{\mathrm{H}}=N_{\mathrm{Gal}}$. Model \#4 has $\alpha_{E}=0.7$ and $N_{\mathrm{H}}$ equal to $N_{\mathrm{Gal}}$ plus $10^{22} \mathrm{~cm}^{-2}$ located at $z_{\mathrm{em}}$. ${ }^{\mathrm{c}}$ Percent error from photon statistics only. Systematic uncertainty from uncertainty in the absolute calibration is about $4 \%$. ${ }^{\mathrm{d}}$ Fit for rp700078. ${ }^{\mathrm{e}}$ Fit for rp700467. function on energy, the choice of radius has a non-negligible effect on the derived $\mathrm{x}$-ray fluxes or spectra. We estimated this effect using PSPC observations of a number of bright low-redshift quasars. We found that the correction on the count rate in the case of a radius of $1.5^{\prime}$ and $2^{\prime}$ is always less than $\sim 5 \%-7 \%$ in the band defined by PI channels $11-40$, and within $1 \%$ in the band defined by PI channels $41-245$ (within $4 \%$ in the whole PI 11-245 band). For a $1^{\prime}$ radius the correction can be significantly larger in the PI 11-41 band. The amplitude of the correction depends strongly on the value of $N_{\mathrm{H}}$. For example, analyzing a ROSAT archive observation of $3 \mathrm{C} 273$, which has galactic $N_{\mathrm{H}}=1.68 \times 10^{20} \mathrm{~cm}^{-2}$ (Savage et al. 1993) and no evidence for intrinsic $N_{\mathrm{H}}$, we found that $21 \%$ of the PI 11-40 photons are outside a $1^{\prime}$ circle $(1.5 \%$ of the PI $41-245$ photons and $9 \%$ of the PI 11-245 photons). Analyzing the observation of NAB0205 +024 , which has galactic $N_{\mathrm{H}}$ of $N_{\mathrm{H}}=3.0 \times 10^{20} \mathrm{~cm}^{-2}$ (Elvis et al. 1989) and a spectrum steeper by $\Delta \alpha_{E}=1$ than that of 3 C273 (Fiore et al. 1994), we found that only $10 \%$ of the PI 11-40 photons are outside a $1^{\prime}$ circle (1\% of the PI $41-245$ photons and $6 \%$ of the PI 11-245 photons). The correction is negligible for $N_{\mathrm{H}}$ higher than $5-7 \times 10^{20} \mathrm{~cm}^{-2}$. We therefore decided to correct for this effect only the count rates of Q0207-398, which has a galactic $N_{\mathrm{H}}$ of $1.53 \times 10^{20} \mathrm{~cm}^{-2}$, similar to that of $3 \mathrm{C} 273$. The count rate was also corrected for dead time, assumed to be $3 \%$. The correction from the exposure mask, which includes corrections for vignetting and for the amount of time the source is under the support wires, is quite small for on-axis sources (smaller than a few percent) and so was neglected when the source was on-axis.

The PSPC fluxes of the quasars are given in Table 3, and are derived from the counts given in Table 2. For most of the quasars, there were too few counts $(\sim 25-70)$ to make the standard two parameter $\mathrm{x}$-ray spectral fits (although see Sec. 2.6). We therefore derived the flux, assuming four different spectral models, which should span the plausible range of expected variations. Models 1,2 , and 3 have the absorption fixed at the galactic value, and $\alpha_{E}=0.5,0.7$, and 1 , respectively. For Model 4, $\alpha_{E}=0.7$, and in addition to the Galactic $N_{\mathrm{H} I}$, absorption at the emission line redshift of $N_{\mathrm{H}}=10^{22}$ $\mathrm{cm}^{-2}$ is added (cf. Paper I). The last column of Table 3 lists the uncertainty from photon statistics. The systematic uncertainty in these quantities is probably about $4 \%$; this is the sum of uncertainties in dead time, exposure time, and flatfielding. The uncertainty from not knowing the source spectrum is comparable to, if not greater than, any other source of error. For comparison, we also list the fluxes derived under these model assumptions for the objects in Paper I, even though those objects do have independent spectral constraints.

The PSPC image of $0130-403$ contains part of the area surveyed for quasars by Hoag \& Smith (1977) and Osmer (1977), and so contains several other quasars with $z \approx 2$ which fall within the PSPC field of view. The x-ray properties of these quasars are given in Tables 4 and 5. Four of the seven quasars within the support ribs were detected. Identification of other serendipitous $\mathrm{X}$-ray sources in this and the other PSPC fields will be discussed in a future paper. 
TABLE 4. Quasars in PSPC image of 0130-403: observed counts.

\begin{tabular}{|c|c|c|c|c|c|c|c|}
\hline Quasar & $z_{e m}$ & V & $\begin{array}{l}\mathrm{RA}(1950) \\
\operatorname{Dec}(1950)\end{array}$ & $\begin{array}{c}\text { Total }^{a} \\
\text { PI 11-245 }\end{array}$ & $\begin{array}{c}\text { Counts } \\
\text { Soft } \\
11-40\end{array}$ & $\begin{array}{c}\text { Hard } \\
41-245\end{array}$ & $\begin{array}{c}\text { Ratio } \\
(\mathrm{H}-\mathrm{S}) / \text { Total }\end{array}$ \\
\hline 0130-401 & 1.73 & 20.3 & $\begin{array}{c}01: 30: 21.2 \\
-40: 10: 09\end{array}$ & $37.1 \pm 9.9$ & $21.4 \pm 8.3$ & $15.7 \pm 5.3$ & $-0.154 \pm 0.269$ \\
\hline $0130-404$ & 2.16 & 19.2 & $\begin{array}{c}01: 30: 45.9 \\
-40: 25: 49\end{array}$ & $29.6 \pm 9.8$ & $13.6 \pm 8.2$ & $16.0 \pm 11.0$ & $0.081 \pm 0.465$ \\
\hline 0131-404 & 1.48 & 19.0 & $\begin{array}{c}01: 31: 03.1 \\
-40: 27: 35\end{array}$ & $<28.8$ & & & \\
\hline $0131-401 \mathrm{~A}$ & 1.83 & 19.8 & $\begin{array}{c}01: 31: 22.1 \\
-40: 11: 16\end{array}$ & $57.5 \pm 11.1$ & $35.9 \pm 9.7$ & $21.6 \pm 6.0$ & $-0.249 \pm 0.204$ \\
\hline $0131-401 \mathrm{C}$ & 1.65 & 20.0 & $\begin{array}{c}01: 31: 29 . \bar{\tau} \\
-40: 08: 15\end{array}$ & $27.8 \pm 9.3$ & $12.1 \pm 7.7$ & $15.7 \pm 5.3$ & $0.129 \pm 0.339$ \\
\hline 0131-402 & 2.11 & 20.3 & $\begin{array}{c}01: 31: 36.6 \\
-40: 17: 56\end{array}$ & $<32.6$ & & & \\
\hline $0132-403$ & 2.18 & 19.5 & $\begin{array}{c}01: 32: 09.6 \\
-40: 18: 08\end{array}$ & $<29.8$ & & & \\
\hline
\end{tabular}

Notes to TABLE 4

${ }^{a}$ Net counts in $2^{\prime}$ circle. Three-sigma upper limits are given when quasar was not detected.

\subsection{The X-ray Spectrum of the Radio-Quiet Quasar $1946+768$}

As discussed below, the radio quiet quasars with $z>3$ in this sample are relatively $x$-ray quiet compared to their lowredshift counterparts, or to radio loud quasars at high redshift. A practical consequence of this is that it is hard to accumulate enough counts in the PSPC to study the $x$-ray spectrum of any radio-quiet quasar at $z>3$; the objects discussed in Paper I for example are all radio loud. In Paper I, we found that there is substantial soft $x$-ray absorption in excess of the galactic $N_{\mathrm{H} I}$ in at least two high-redshift radioloud quasars. However, the origin of the absorbing material is not clear since the absorption redshift is not measurable from the PSPC data. If substantial $x$-ray absorption were detected for even one radio-quiet quasar at $z>3$, it would be strong evidence that such absorption is not related solely to radio loudness.

The discovery of $1946+768$ (Hagen et al. 1992), which is radio quiet (Kuhn et al. 1994) and very luminous $(V=15.8$ and $z=3.02$ ), seemed to present a good opportunity to study the $\mathrm{x}$-ray spectrum of a high redshift, radio-quiet quasar. Unfortunately, it is in the direction of a relatively large column

TABLE 5. X-ray properties of quasars in PSPC image of $0130-403$.

\begin{tabular}{lcccccccc}
\hline \hline Quasar & $z_{e m}$ & $\begin{array}{c}\mathrm{t}_{\text {live }}{ }^{a} \\
\text { seconds }\end{array}$ & $\mathrm{f}_{x}{ }^{b}$ & $\begin{array}{c}\% \\
\text { Error }^{c}\end{array}$ & $\mathrm{f}_{\text {opt }}{ }^{d}$ & $1_{x}{ }^{c}$ & $1_{\text {opt }}{ }^{f}$ & $\alpha_{\text {ox }}{ }^{g}$ \\
\hline $0130-401$ & 1.73 & 5793 & 2.59 & 27 & -4.396 & 27.519 & 30.819 & 1.267 \\
$0130-404$ & 2.16 & 5732 & 2.09 & 33 & -3.956 & 27.680 & 31.484 & 1.460 \\
$0131-404$ & 1.48 & 5665 & $<1.97$ & - & -3.876 & $<27.223$ & 31.185 & $>1.521$ \\
$0131-401 \mathrm{~A}$ & 1.83 & 5712 & 4.08 & - & -4.196 & 27.938 & 31.076 & 1.205 \\
$0131-401 \mathrm{C}$ & 1.65 & 5500 & 2.04 & 33 & -4.276 & 27.390 & 30.892 & 1.344 \\
$0131-402$ & 2.11 & 5642 & $<1.20$ & - & -4.396 & $<27.413$ & 31.020 & $>1.385$ \\
$0132-403$ & 2.18 & 4485 & $<1.38$ & - & -4.076 & $<27.452$ & 31.373 & $>1.505$ \\
\hline
\end{tabular}

Notes to TABLE 5

${ }^{a}$ Effective exposure time, estimated from exposure map, including dead time correction $3 \%$. ${ }^{\mathrm{b}} \mathrm{X}$-ray flux at observed energy of $1 \mathrm{keV}$ in $10^{-5}$ photons $\mathrm{cm}^{-2} \mathrm{~s}^{-1} \mathrm{keV}^{-1}$, derived from total counts in pha bins 11-245, assuming spectral index of $\alpha_{E}=0.5$, and $N_{\mathrm{H}}$ equal to the galactic value. ${ }^{c}$ Percent error, from photon statistics only. ${ }^{d} \log$ of observed flux at rest wavelength of $2500 \AA$, in $\mathrm{Jy}$. ${ }^{\mathrm{e}} \log$ of monochromatic luminosity at rest energy of $2 \mathrm{keV}$, assuming $\alpha_{E}=0.5$, in erg s $\mathrm{sz}^{-1} \mathrm{~Hz}^{-1} \cdot H_{0}=50 \mathrm{~km} \mathrm{~s}^{-1} \mathrm{Mpc}^{-1}$ and $q_{0}=0$ were assumed. $\log$ of monochromatic luminosity at rest wavelength of $2500 \AA$, derived from $m(1450)$, assuming $\alpha_{\text {opt }}=0.7$; units and cosmology as in (e). ${ }^{\mathrm{g}} \alpha_{\mathrm{ox}}$; see Eq. (5) in text. of galactic $\mathrm{H} \mathrm{I}, N_{\mathrm{H} I}=7.5 \times 10^{20} \mathrm{~cm}^{-2}$, and turns out to be even more $x$-ray quiet than we predicted. In any event, $95 \pm 11$ counts were detected, and the spectral fits are summarized in Table 6. The fitting procedures were identical to those described in Paper I.

The PSPC data are consistent with a power law with energy index $\alpha_{E}=1.8_{-1.4}^{+2.1}$, with no evidence of absorption in addition to the galactic column and a $\chi^{2}$ of 1.00 with 4 degrees of freedom $\left(\alpha_{E}=1.00_{-0.32}^{+0.28}\right.$ with an acceptable $\chi_{r}^{2}=1.2$ with 5 degrees of freedom, see Table 6). A spectral fit with local absorption fixed at the galactic value plus free absorption at $z=3.02$ yields an intrinsic $N_{\mathrm{H}}$ of $\left(15.1_{-15.1}^{+44.7}\right) \times 10^{20}$ $\mathrm{cm}^{-2}$, with a three $3 \sigma$ upper limit of intrinsic $N_{\mathrm{H}}<1.3 \times 10^{22}$ $\mathrm{cm}^{-2}$. In comparison, the $\mathrm{x}$-ray absorption detected for 2126 -158 has $N_{\mathrm{H}}=1.4 \times 10^{22} \mathrm{~cm}^{-2}$ and for $0438-436$ has $N_{\mathrm{H}}=0.86 \times 10^{22} \mathrm{~cm}^{-2}$ (Paper I). So the upper limit for $N_{\mathrm{H}}$ for $1946+768$ is just barely sensitive enough to rule out absorption at the level detected for $0438-436$ and 2126-158 (Paper I). Figure 2 shows the PSPC pulse height spectrum for $1946+768$ and the best fit power-law model.

We note that the program GETNH in XANADU gives a somewhat higher value of the galactic column for this object, $N_{\mathrm{H} I}=9.28 \times 10^{20} \mathrm{~cm}^{-2}$. Examination of the Stark et al. (1989) H I maps, reveals that the lower value is likely correct. However, for comparison, we give the spectral fits for both values of $N_{\mathrm{H} I}$ in Table 6 . The uncertainty in the $N_{\mathrm{H} I}$ does not change the $\mathrm{x}$-ray spectral parameters significantly.

\subsection{The X-ray Spectrum of 0000-263}

Our observation of 0000-263 yielded 26 net counts in $4300 \mathrm{~s}$. A much longer exposure was later obtained by $\mathrm{M}-\mathrm{H}$. Ulrich (observation rp700467) and became available in the ROSAT archive. This exposure yielded 162 net photons in $37420 \mathrm{~s}$. Spectral fits to the PSPC data are summarized in Table 6, and the fluxes and colors from the longer exposure are given in Tables 2 and 3. The fluxes, x-ray colors, and spectral properties derived from the longer exposure agree with those obtained in our data.

The power law energy index is $\alpha_{E}=2.0_{-1.4}^{+0.7}$, with little evidence of absorption in addition to the galactic one and $\chi^{2}=0.82$ with 7 degrees of freedom $\left(\alpha_{E}=1.30 \pm 0.23\right.$ with an acceptable $\chi_{r}^{2}=0.78$ with 8 degrees of freedom, see Table 6 ). We tried also a model which included absorption at the emission line redshift, $z_{\mathrm{em}}=4.11$. The $3 \sigma$ upper limit is $N_{\mathrm{H}}<5.6 \times 10^{22} \mathrm{~cm}^{-2}$.

This limit is less strong than that derived for $1946+776$, since the redshift of $0000-263$ is higher, and the soft absorption is shifted farther out of the PSPC band. Thus, a higher column is necessary to have detectable absorption. Since the $3 \sigma$ limit on $N_{\mathrm{H}}$ for $0000-263$ is somewhat higher than the $N_{\mathrm{H}}$ measured for the objects with detected absorption (2126 -158 and $0438-436$ ), these observations have not ruled out the presence of comparable absorption in this object.

\subsection{The X-ray Spectrum of $0014+813$}

In Paper I, we discussed the spectral model fits to the PSPC data for $0014+813$. This quasar was also observed with the EXOSAT ME and its spectrum in the energy range 
TABLE 6. X-ray spectral fits.

\begin{tabular}{|c|c|c|c|c|c|}
\hline QSO & fit & Flux $^{a}$ & $a_{E}$ & $N_{H}^{b}$ & $\backslash_{\nu}^{2}($ dof $)$ \\
\hline \multicolumn{6}{|l|}{$1946+768^{\circ}$} \\
\hline PSPC & 1 & $0.485 \pm .20$ & $1.80_{-1.4}^{+2.1}$ & $15_{-17.6}^{+44.7}$ & $1.00(4)$ \\
\hline PSPC & 2 & $0.381 \pm 0.043$ & $1.00_{-0.32}^{+0.28}$ & T.5 (Fixed) & $1.19(5)$ \\
\hline PSPC & 3 & $0.391 \pm 0.045$ & $0.97 \pm 0.36$ & 9.3 (Fixed) & $1.69(6)$ \\
\hline \multicolumn{6}{|l|}{$0000-263^{d}$} \\
\hline PSPC & 1 & $0.099_{-0.034}^{+0.030}$ & $2.04_{-1.4}^{+0.71}$ & $382_{-270}^{+500}$ & $0.82(\tau)$ \\
\hline PSPC & 2 & $0.091 \pm 0.040$ & $1.30 \pm 0.23$ & 1.6(Fixed) & $0.78(8)$ \\
\hline PSPC & 3 & $0.100 \pm 0.030$ & $2.00_{-1.0}^{+1.5}$ & $80_{-70}^{+160}$ & $0.80(\tau)$ \\
\hline \multicolumn{6}{|l|}{$0014+813^{e}$} \\
\hline PSPC & 1 & $5.5_{-1.1}^{+2.6}$ & $1.0 i_{-0.73}^{+1.2}$ & $19_{-8}^{+24}$ & $1.06(18)$ \\
\hline PSPC & 2 & $4.7 \pm 0.27$ & $0.82 \pm 0.19$ & 14.4 (Fixed) & $1.02(19)$ \\
\hline PSPC & 3 & $5.05_{-0.6}^{+2.0}$ & $0.92 \pm 0.67$ & $64_{-64}^{+500}$ & $1.07(18)$ \\
\hline EXOSAT ME & 4 & $4.54_{-1.77}^{+2.73}$ & $0.58_{-0.34}^{+0.35}$ & 14.4 (Fixed) & $1.05(62)$ \\
\hline EXOSAT ME & 5 & $4.83_{-0.31}^{+2.49}$ & 0.63 (Fixed) & 14.4 (Fixed) & $1.03(63)$ \\
\hline EXOSAT ME + PSPC & 6 & $4.41_{-0.66}^{+1.03}$ & $0.58_{-0.18}^{+0.22}$ & $11.9_{-4.3}^{+7.4}$ & $1.04(82)$ \\
\hline \multicolumn{6}{|l|}{$2126-158^{f}$} \\
\hline PSPC & 1 & $10.4_{-1.6}^{+3.9}$ & $0.54 \pm 0.44$ & $11_{-4}^{+11}$ & $1.37(20)$ \\
\hline PSPC & 2 & $8.2 \pm 0.3$ & $-0.06 \pm 0.11$ & 4.85 (Fixed) & $1.71(21)$ \\
\hline PSPC & 3 & $9.5_{-0.9}^{+1.4}$ & $0.37_{-0.31}^{+0.40}$ & $120_{-75}^{+200}$ & $1.39(20)$ \\
\hline EXOSAT ME & 4 & $13.0_{-4.0}^{+5.9}$ & $0.41 \pm 0.25$ & 120 (Fixed) & $0.96(30)$ \\
\hline EXOSAT ME & 5 & $15.3 \pm 0.11$ & 0.37 (Fixed) & 120 (Fixed) & $0.94(30)$ \\
\hline $\operatorname{EXOSAT~ME~}+\operatorname{LE}(6+\bar{\imath})$ & 6 & $12.5_{-0.6}^{+1.1}$ & $0.38_{-0.40}^{+0.45}$ & $8.6_{-0.7}^{+2.4}$ & $0.94(31)$ \\
\hline EXOSAT ME + LE $(6+i)$ & $i$ & $14.0_{-1.7}^{+1.6}$ & $0.45_{-0.10}^{+0.09}$ & 120(Fixed) & $0.91(32)$ \\
\hline EXOSAT ME $+\operatorname{LE}(6+7)$ & 8 & $14.9 \pm 1.0$ & 0.37 (Fixed) & 120(Fixed) & $0.90(33)$ \\
\hline
\end{tabular}

Notes to TABLE 6

${ }^{a}$ Flux at $1 \mathrm{keV}$, in units of $10^{-4}$ photons $\mathrm{cm}^{-2} \mathrm{~s}^{-1} \mathrm{keV}^{-1} \cdot{ }^{\mathrm{b}} N_{\mathrm{H}}$ in units of $10^{20} \mathrm{~cm}^{-2}$. ${ }^{\mathrm{c}}$ For $1946+768$, Fit: $1-z=0 N_{\mathrm{H}}$ free; $2-z=0 N_{\mathrm{H}}$ fixed at the galactic $\mathrm{H}$ I value, for $N(\mathrm{H} \mathrm{I})=7.5 \times 10^{20} \mathrm{~cm}^{-2} ; 3-z=0 N_{\mathrm{H}}$ fixed at the galactic $\mathrm{H}$ I value, for $N(\mathrm{H} \mathrm{I})=9.3 \times 10^{20} \mathrm{~cm}^{-2}$. ${ }^{\mathrm{d}} \mathrm{For} 0000-263$, Fit: $1-z=0 N_{\mathrm{H}}$ and $\alpha_{E}$ free; $2-z=0 N_{\mathrm{H}}$ fixed at the galactic $\mathrm{H}$ I value; 3 -absorber in the quasar frame, plus absorption fixed at the galactic $\mathrm{H} \mathrm{I} \mathrm{value.}{ }^{\mathrm{e}} \mathrm{For} 0014+813$, PSPC fits are from Paper I. Fit: $1-z=0 N_{\mathrm{H}}$ free; $2-z=0 N_{\mathrm{H}}$ fixed at the galactic $\mathrm{H}$ I value; 3 -absorber in the quasar frame, plus absorption fixed at the galactic $\mathrm{H}$ I value; ${ }^{\mathrm{f}}$ For 2126-158, PSPC fits are from Paper I. Fit: $1-z=0 N_{\mathrm{H}}$ free; $2-z=0 N_{\mathrm{H}}$ fixed at the galactic $\mathrm{H}$ I value; 3 -absorber in the quasar frame, plus absorption fixed at the galactic $\mathrm{H}$ I value; $4-\mathrm{ME}$ data alone, absorption fixed to be the PSPC value given in fit 3; 5-ME data, absorption and spectral index fixed at the PSPC values given in fit 3;6-ME and LE data, absorption at $z=0$ and $\alpha_{E}$ free; 7-ME and LE data, absorption fixed at PSPC values given in fit 3; 8-ME and LE data, absorption and spectral index fixed at PSPC values given in fit 3.

1.4-6 keV was discussed by Bierman et al. (1992) and Lawson et al. (1992). Unfortunately, neither of these papers gave the flux normalization of the best power law fits to the data, so direct comparison to the PSPC results was not possible. We therefore obtained the EXOSAT ME data from the HEASARC.

The results of the spectral fits are given in Table 6 . The galactic $N_{\mathrm{H} I}$ for this object is very high, so the limits on intrinsic or intervening absorption are not very strong. However, the EXOSAT data are consistent in both spectral index and flux to the PSPC results. A simultaneous fit to the EXOSAT ME and PSPC data gives $\alpha_{E}=0.58_{-0.18}^{+0.22}$ and an $N_{\mathrm{H}}$ consistent with the galactic $N_{\mathrm{H} I}$. The observed energy range is 0.1 to $6 \mathrm{keV}$, corresponding to 0.44 to $26.5 \mathrm{keV}$ in the rest frame.

\subsection{The $X$-ray Spectrum of $2126-158$}

The PSPC spectrum of this quasar was discussed in Paper I. It was also observed with EXOSAT in 1984 (day 134). The EXOSAT data has been discussed by McGlynn et al. (1985) and Ghosh \& Soundararajaperumal (1992). In order to see if our PSPC spectral fits were consistent with the EXOSAT data, we obtained the ME data from the HEASARC, which had an exposure time of $30050 \mathrm{~s}$, and a total count rate of $0.52 \pm 0.05$ counts $\mathrm{s}^{-1}$ in the 1 to $8 \mathrm{keV}$ range. The quality of the spectrum is poor, "array swaps" (i.e., chopping) were not performed, and the background subtraction was done using a slew (Smith 1984). There were also two LE exposures. One was with the lexan filter (filter 7) and had an exposure time of $17451 \mathrm{~s}$ and a count rate of $4.0 \pm 0.7 \times 10^{-3}$ counts $\mathrm{s}^{-1}$. The other was with the aluminum-parylene filter (filter 6), for $20500 \mathrm{~s}$, and a net count rate of $2.6 \pm 0.6 \times 10^{-3}$ counts $\mathrm{s}^{-1}$

We have refit the EXOSAT data, and the results are given in Table 6. The results of the fits to the PSPC data from Paper I are also listed for comparison. We tried fitting the ME data alone, and the ME data with the LE data with both $\alpha_{E}$ and $N_{\mathrm{H}}$ free; these spectral constraints are not very good. Fixing the absorption and power law index to the best fit PSPC value gives an acceptable fit to the EXOSAT data (fit 5 in Table 6). Thus, the spectral shape of the EXOSAT data is consistent with the PSPC results, although the flux at $1 \mathrm{keV}$ is marginally higher (by $\sim 30 \%$ at $3 \sigma$ confidence level). However, because of the poor quality of the ME spectrum, this evidence for $\mathrm{x}$-ray variability in $2126-158$ is weak.

\subsection{Constraints on X-ray Spectra from PSPC Colors}

In the cases where fewer than $\sim 100$ photons were detected in the PSPC, there are not enough counts to fit x-ray spectral models. However, some limited spectral information can be derived from an X-ray color, or hardness ratio. Maccacaro et al. (1988) used an IPC hardness ratio to derive a 


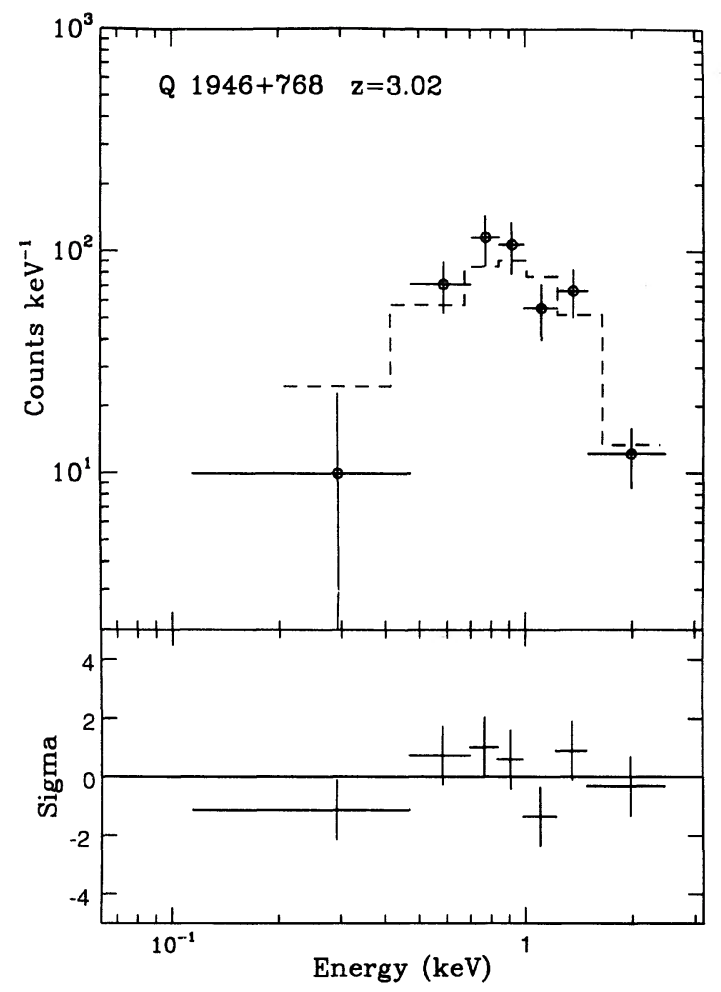

FIG. 2. ROSAT PSPC pulse height spectra for $1946+768$. The upper panel shows the pulse height spectrum and best fit power-law plus absorption model (fit 1 of Table 6), and the lower panel shows the residuals from the fit.

mean spectral index for the Einstein Medium Sensitivity Survey AGN and quasars. They found $\left\langle\alpha_{E}\right\rangle=1.03$ in the 0.3 to $3.5 \mathrm{keV}$ band, assuming the total absorption is limited to the galactic column.

In Table 2 we list the hardness ratios for the quasars detected in the PSPC, defined as

$$
R=(H-S) /(H+S) .
$$

Here $H$ is the number of counts in a hard band defined as PI channels $41-245$, or 0.41 to $2.48 \mathrm{keV}$ in the observed frame; $S$ is the number of counts in a soft band defined as PI channels 11-40, or observed energies of 0.11 to $0.40 \mathrm{keV}$. Note that for an object at $z=3$, these channels correspond to 0.44 $1.64 \mathrm{keV}$ and $1.64-9.92 \mathrm{keV}$ in the rest frame, respectively.

In order to interpret these ratios, predicted model spectra were generated using the SINGLEFIT package in PROS. Figure 3 shows the result for one of the objects, 0000-263. The hardness ratio is plotted as a function of spectral index, $\alpha_{E}$, for several models for the absorption by $N_{\mathrm{H}}$. For model (a), $N_{\mathrm{H}}$ was assumed to be equal to the galactic $N_{\mathrm{H} I}$ value only; for model (b), an absorber with $\log N_{\mathrm{H}}=21.5 \mathrm{~cm}^{-2}$ at $z_{\mathrm{em}}$ was added. For models (c) $-(\mathrm{e})$ absorbers with $\log N_{\mathrm{H}}=22.0$, 22.5 , and $23.0 \mathrm{~cm}^{-2}$ at $z_{\mathrm{em}}$ were added. In all cases the absorbers were assumed to have cross sections given by Morrison \& McCammon (1983) with solar metal abundance.

If we assume there is no absorption in excess of the Milky Way $\mathrm{H}$ I column, then from Fig. 3 the spectral index for 0000-263 is remarkably well constrained even with our

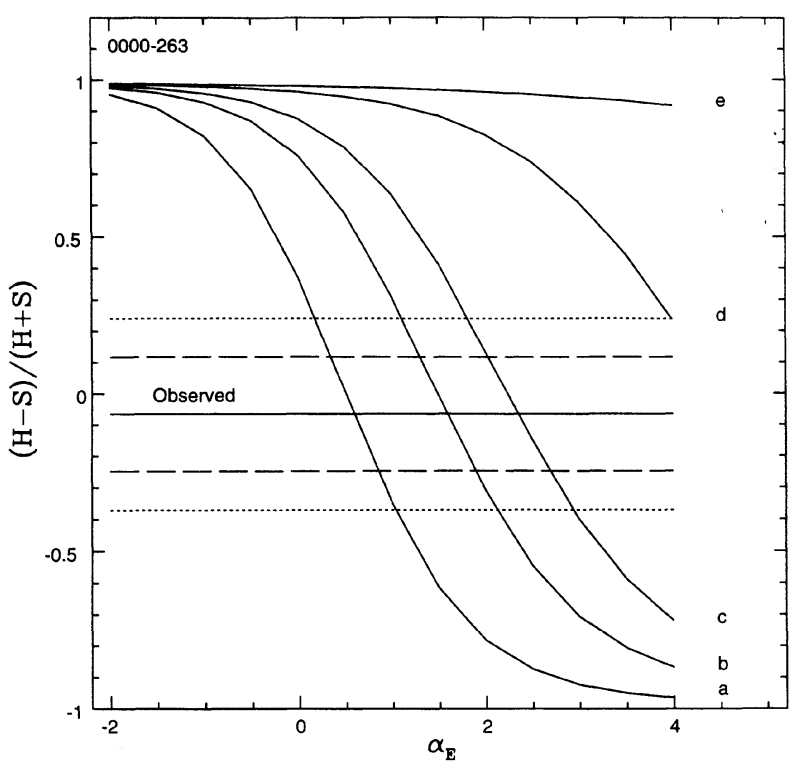

FIG. 3. For $0000-263$, the PSPC $x$-ray hardness ratio as a function of power law energy spectral index, $\alpha_{E}$. Curve a: Absorption assumed to be galactic only, $\log N_{\mathrm{Gal}}=19.477 \mathrm{~cm}^{-2}$. Curve b: Absorption assumed to be galactic plus $\log N_{\mathrm{H}}=21.5 \mathrm{~cm}^{-2}$ at $z=4.11$. Curve c: Absorption assumed to be galactic plus $\log N_{\mathrm{H}}=22.0 \mathrm{~cm}^{-2}$ at $z=4.11$. Curve d: Absorption assumed to be galactic plus $\log N_{\mathrm{H}}=22.5 \mathrm{~cm}^{-2}$ at $z=4.11$. Curve e: Absorption assumed to be galactic plus $\log N_{\mathrm{H}}=23.0 \mathrm{~cm}^{-2}$ at $z=4.11$. Solid line is the observed hardness ratio, dashed lines indicate $3 \sigma$ error from all data, dotted lines indicate $3 \sigma$ error from short exposure only.

short exposure (dotted lines in Fig. 3), where we detected only 26 net photons. The hardness ratio gives $\alpha_{E}=1.22(+0.49,-0.47)$, in reasonable agreement with the long exposure spectral fit result, $\alpha_{E}=1.30 \pm 0.23$.

We carried out a similar analysis for all the program objects which were detected, and the results are shown in Fig. 4 and summarized in Table 7. For comparison, we computed $\alpha_{E}$ with the absorption column fixed at the galactic value for the objects that had spectral fits (Paper I). The comparison between the $\alpha_{E}$ derived from spectral fitting and from the hardness ratio (assuming in both cases that the absorption is fixed at the galactic value) is plotted in Fig. 5. For the objects where x-ray absorption is detected (0438-436 and 2126 -158) the agreement between the $\alpha_{E}$ derived from the hardness ratio assuming no absorption and the spectral fit values is not good, but for the other objects the agreement is adequate.

The results for $\alpha_{E}$ for the objects without sufficient counts to make spectral fits are shown in Fig. 6. For $0636+68$, the hardness ratio is $R>1$, so only an upper limit of $\alpha_{E}<0.39$ $(1 \sigma)$ can be placed. The weighted mean of the spectral indices, calculated using the hardness ratios, for the radio quiet objects $1208+101,1107+481,0207-398$, and $0130-403$ is $\left\langle\alpha_{E}\right\rangle=1.11 \pm 0.21$.

The weighted mean of the spectral indices of all radio quiet objects, including the spectral indices of Q0000-263 and Q1946 +768 given in Table 6 , is $\left\langle\alpha_{E}\right\rangle=1.15 \pm 0.14$. Note that this spectral index is for $0.42-9.4 \mathrm{keV}$ for the lowest 

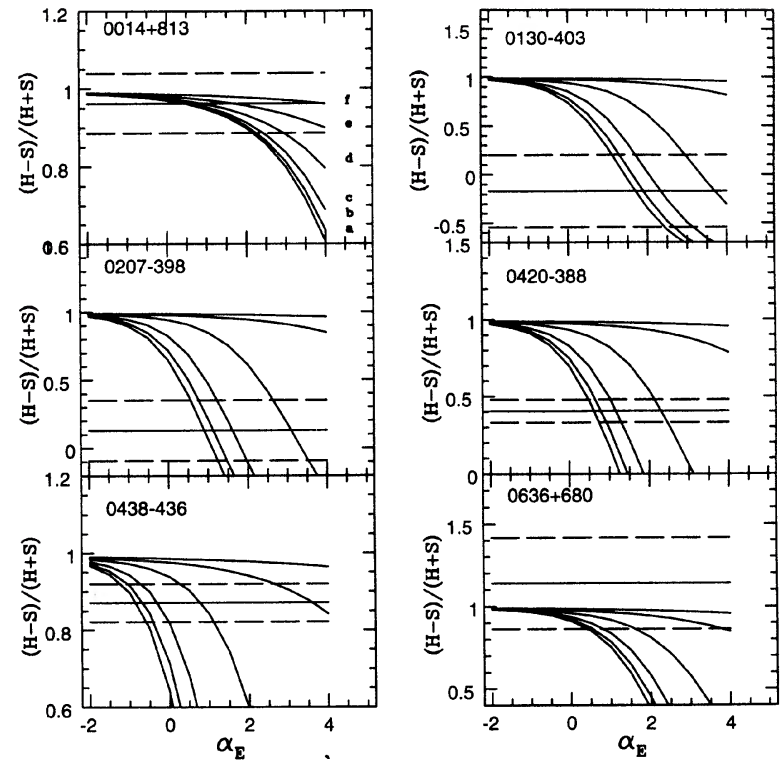

TABLE 7. X-ray spectral constraints from PSPC colors.

\begin{tabular}{lcccc}
\hline \hline Quasar & $z_{e m}$ & $\begin{array}{c}\alpha_{E^{a}} \\
\mathrm{~N}_{H} \equiv \text { Galactic }\end{array}$ & $\begin{array}{c}\mathrm{N}_{H}{ }^{b} \\
\alpha_{E} \equiv 0.7\end{array}$ & $\begin{array}{c}\alpha_{E^{c}} \\
\mathrm{~N}_{H}=10^{22} \text { at } \mathrm{z}_{e m}\end{array}$ \\
\hline $0000-263^{d}$ & 4.11 & $1.22(+0.49,-0.47)$ & $<195$ & $2.35(+0.61,-0.50)$ \\
$0000-263^{e}$ & 4.11 & $1.22(+0.29,-0.29)$ & $<32$ & $2.35(+0.32,-0.33)$ \\
$0014+813$ & 3.38 & $0.182(+1.88, f)$ & & \\
$0130-403$ & 3.03 & $1.69(+0.71,-.60)$ & $<251$ & $2.30(+0.80,-0.55)$ \\
$0207-398^{e}$ & 2.81 & $0.88(+0.35,-0.33)$ & $<56$ & $3.09(+.43,-0.43)$ \\
$0420-388$ & 3.12 & $0.59(+0.15,-0.11)$ & & \\
$0438-436^{e}$ & 2.85 & $-0.87(+0.24,-0.33)$ & & \\
$0636+680$ & 3.17 & $<0.39 f$ & $b$ & $<1.61^{f}$ \\
$1107+481$ & 2.96 & $0.93(+0.39,-0.38)$ & $<89$ & $1.61(+0.39,-0.48)$ \\
$1208+101$ & 3.82 & $1.54(+0.73,-0.53)$ & $<112$ & $2.05(+0.70,-0.60)$ \\
$1946+768$ & 3.02 & $0.42(+1.41, f)$ & $f$ & $0.60(+1.6, f)$ \\
$2000-330$ & 3.78 & $1.61(+0.71,-0.92)$ & $f$ & $2.55\left(+0.85,{ }^{f}\right)$ \\
$2126-158^{e}$ & 3.27 & $-1.25(+0.85, f)$ & & \\
$0130-401$ & 1.73 & $1.66(+0.51,-0.44)$ & $<12$ & $6.20(+0.73,-0.70)$ \\
$0130-404$ & 2.16 & $1.27(+0.79,-0.80)$ & $f$ & $4.49(+1.16,-1.12)$ \\
$0131-401 \mathrm{~A}$ & 1.83 & $1.83(+0.38,-0.36)$ & $<2.3$ & $6.16(+0.57,-0.59)$ \\
$0131-401 \mathrm{C}$ & 1.65 & $1.20(+0.53,-0.59)$ & $f$ & $5.72(+0.90,-0.89)$ \\
\hline & & & &
\end{tabular}

Notes to TABLE 7

${ }^{a} \alpha_{E}$ assuming $N_{\mathrm{H}}$ equal to the galactic $N_{\mathrm{H},} ; 1 \sigma$ error is given. ${ }^{\text {b }}$ Three sigma upper limit to $N_{\mathrm{H}}$ in units of $10^{20} \mathrm{~cm}^{-2}$, assuming a power law, and $\alpha_{E}=0.7$. For $0636+680$, the predicted color for $\alpha_{E}=0.7$ is $>0.80$ for any $N_{\mathrm{H}}$ and therefore no limit is derivable. ${ }^{\mathrm{c}} \alpha_{E}$ assuming $N_{\mathrm{H}}=10^{22} \mathrm{~cm}^{-2}$ at $Z_{\mathrm{em}}$ in addition to the galactic $N_{\mathrm{H}} ; 1 \sigma$ error is given. ${ }^{\mathrm{d}}$ Data of November 1991 only. ${ }^{\mathrm{e}}$ All data summed together. ${ }^{\mathrm{f}}$ Hardness ratio $>1$ for values not listed. For
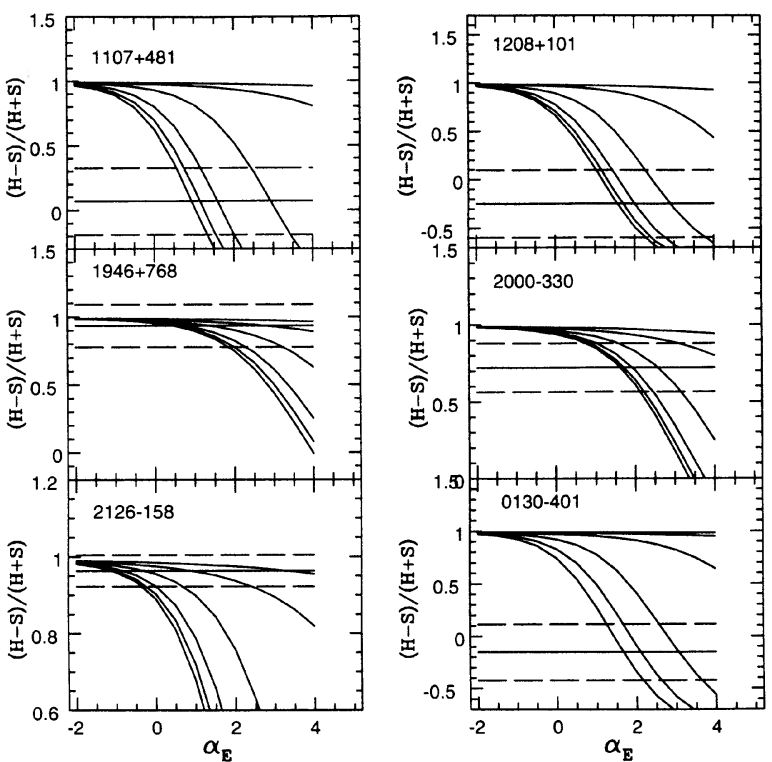
$0636+680,1 \sigma$ upper limit is listed.

redshift object at $z=2.81$, and $0.56-12.7 \mathrm{keV}$ for the highest redshift object at $z=4.11$.

We have carried out the same analysis for the intermediate redshift quasars in the 0130-403 PSPC field. The results are tabulated in Table 7. For these quasars, the $\alpha_{E}$ 's are slightly steeper than the higher redshift objects: the weighted mean is $\left\langle\alpha_{E}\right\rangle=1.61 \pm 0.26$. This is not the result of the objects being off-axis. There is little change in $\alpha_{E}$ derived from colors for sources within the ribs (Ciliegi et al. 1994). However, the steepness of the $\mathrm{x}$-ray spectra of these objects may be related
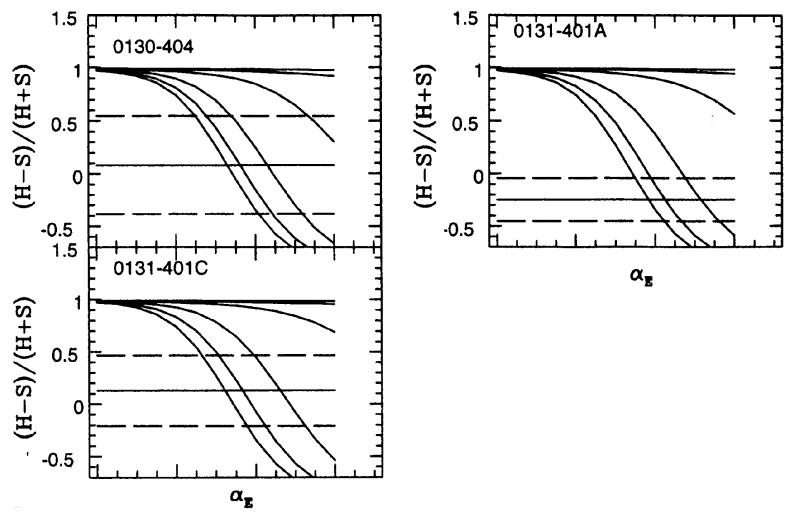

FIG. 4. For the other quasars in the sample, the same plot as Fig. 3, except the redshifted absorbers are assumed to be at the redshift of the quasar shown.

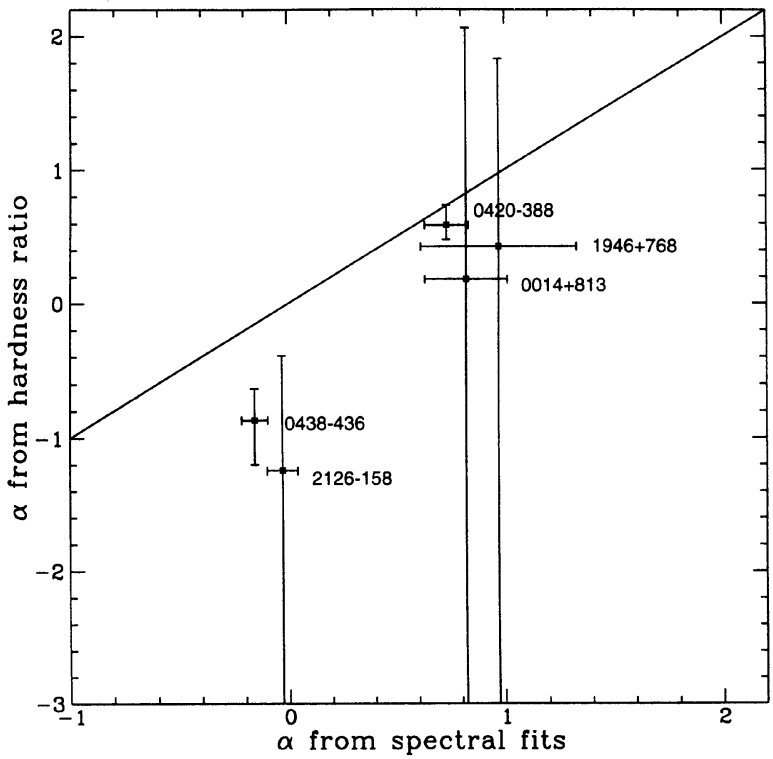

FIG. 5. For quasars with PSPC spectral fits, comparison of $\alpha_{E}$ from spectral fits to $\alpha_{E}$ derived from the hardness ratio. In both cases, the absorption is fixed at the galactic value. Solid line indicates equality. 


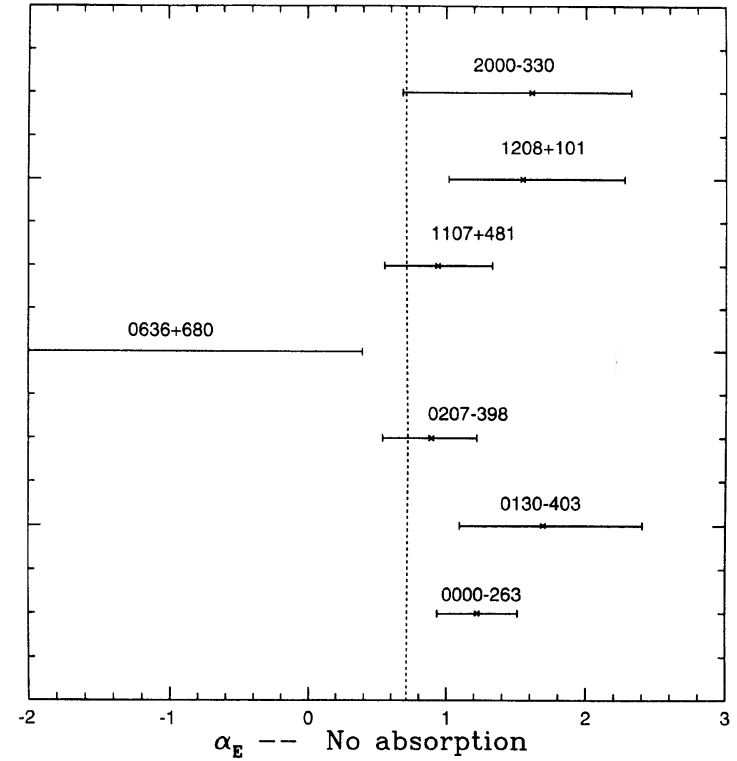

Fig. 6. For quasars without X-ray spectral fits, $\alpha_{E}$ derived from the hardness ratio assuming the absorption is fixed at the galactic value. One sigma range is shown. For $0636+680$, the hardness ratio is greater than 1 , so only an upper limit to $\alpha_{E}$ can be placed. The dotted line indicates $\alpha_{E}=0.7$.

to the energy band observed. For these quasars, the PSPC bandpass corresponds to $\sim 0.3-7 \mathrm{keV}$, between the harder $\mathrm{x}$-rays observed by Ginga and the softer x-rays observed by the IPC for low redshift objects.

These results are discussed in more detail by Bechtold et al. (1994). There we argue that the hardness ratios imply that the x-ray spectra of radio-loud and radio-quiet quasars evolve differently with redshift. Compared to low redshift quasars, the radio quiet quasars have large $N_{\mathrm{H}}$ and steeper $\alpha_{E}$ 's or no absorption and similar $\alpha_{E}$ 's. On the other hand, the radio loud quasars at high redshift appear to have substantial absorption and similar $\alpha_{E}$ 's to low redshift quasars.

\section{NEAR-INFRARED AND OPTICAL SPECTROSCOPY}

Optical spectrophotometry of the program objects was obtained at the Steward Observatory $2.3 \mathrm{~m}$ telescope with the Boller \& Chivens Spectrograph and TI CCD. Details are given in Table 8 . A $3001 / \mathrm{mm}$ grating was used in first order with a 4.5 arcsec wide and 3 arcmin long slit, resulting in resolution of $15 \AA$ (FWHM). Second-order light was eliminated by an order-blocking filter when appropriate. Quasars were observed for a maximum exposure of $3000 \mathrm{~s}$, with the slit oriented at the average parallactic angle for each exposure. For the flux calibration, an IIDS standard star nearby in the sky was observed immediately before and after each quasar observation. Bias frames, quartz-lamp flats and heliumneon-argon lamp exposures were used to calibrate the data in the standard way. The data were reduced in IRAF, and the default standard star flux values and extinction curves for Kitt Peak given by IRAF were used. All data were obtained in photometric skies.
TABLE 8. Log of optical and near-IR spectrophotometry.

\begin{tabular}{lcccc}
\hline \hline Quasar & $z_{e m}$ & Date & $\operatorname{Exp}^{a}(\mathrm{sec})$ & Wavelength Range \\
\hline $0000-263$ & \multirow{2}{*}{1.11} & $12 / 12 / 89$ & $300 \times 10$ & $0.95-1.3 \mu$ \\
& & $11 / 2 / 89$ & 6000 & $4500-7500 \AA$ \\
& & $11 / 4 / 89$ & 6000 & $5500-8500 \AA$ \\
$0014+813$ & 3.38 & $12 / 12 / 89$ & $300-480 \times 9$ & $0.95-1.3 \mu$ \\
& & $11 / 2 / 89$ & $i 200$ & $4500-7500 \AA$ \\
$0636+680$ & 3.17 & $11 / 4 / 89$ & 3000 & $3500-6500 \AA$ \\
& & $11 / 12 / 89$ & $420 \times 10$ & $0.95-1.3 \mu$ \\
$1107+481$ & 2.96 & $4 / 14-15 / 99$ & 4800 & $3500-6500 \AA$ \\
& & $5 / 21 / 90$ & 3000 & $4500-7500 \AA$ \\
$1159+123$ & 3.53 & $4 / 13 / 90$ & $300 \times 10$ & $0.95-1.3 \mu$ \\
& & $5 / 21 / 90$ & 3000 & $4500-7500 \AA$ \\
$1208+101$ & 3.82 & $4 / 13-14 / 90$ & $300 \times 10$ & $0.95-1.3 \mu$ \\
& & $5 / 21 / 90$ & 3000 & $4500-7500 \AA$ \\
$2000-330$ & 3.78 & $11 / 2.4 / 89$ & 4800 & $4500-7500 \AA$ \\
$2126-158$ & 3.28 & $11 / 3-4 / 89$ & $i 200$ & $3500-7500 \AA$ \\
& & $\tau / \tau / 89$ & 1800 & $3500-6500 \AA$ \\
\hline
\end{tabular}

Notes to TABLE 8

${ }^{a}$ For IR spectroscopy, exposure time per grating setting in seconds, and number of grating settings used.

Spectrophotometry in the range $0.9-1.4 \mu \mathrm{m}$ was obtained at the Multiple Mirror Telescope (MMT) with the Germanium Spectrometer. Details are given in Table 8 . The detector was a $2 \times 32$ array of Ge photodiodes from Ford Aerospace (Rieke et al. 1987). A $150 \mathrm{l} / \mathrm{mm}$ grating in first order was used, with double 3 " apertures separated by $22^{\prime \prime}$ resulting in resolution of $\Delta \lambda / \lambda \approx 300-400$, depending on wavelength. At each grating setting, the wavelength coverage was about 0.1 $\mu \mathrm{m}$, so about ten exposures at overlapping grating settings were obtained for each object. These exposures were interlaced with exposures on bright A, F, or G stars, used for flux calibration. While the exposure time at each grating setting was short (5-10 $\mathrm{min})$, it took several hours to obtain the complete spectrum of each object. The individual scans were spliced together, and are shown with the optical data in Fig. 7. The optical and IR data agree in absolute level.

\subsection{Corrections for Reddening and Absorption}

The spectral energy distributions should be corrected for reddening by dust in the Milky Way. If we use the relation

$$
N(\mathrm{H} \mathrm{I}) / \mathrm{E}(B-V)=4.8 \times 10^{21} \text { atoms cm }{ }^{-2} \mathrm{mag}^{-1}
$$

from Bohlin (1978), and the reddening law of Savage \& Mathis (1979) and Rieke \& Lebofsky (1985), the correction in the observed optical and IR is small for $\mathrm{E}(B-V)<0.03$. The effect is shown for $0000-263$ in Fig. 8, where the observed spectrum is plotted as a dotted line, and the spectrum corrected for galactic reddening is shown as curve "a." Galactic extinction was thus neglected in all objects except $0014+813$. For $0014+813$, the galactic column is large, so the correction for galactic reddening is significant: $\mathrm{E}(B-V)$ $=0.30$ assuming the relation of Eq. (3). Figure 9 shows the spectrum of $0014+813$ as observed (curve "a"), and corrected for galactic extinction (curve " $b$ ").

The spectrophotometry was obtained through a wide slit so that corrections for atmospheric refraction and seeing 

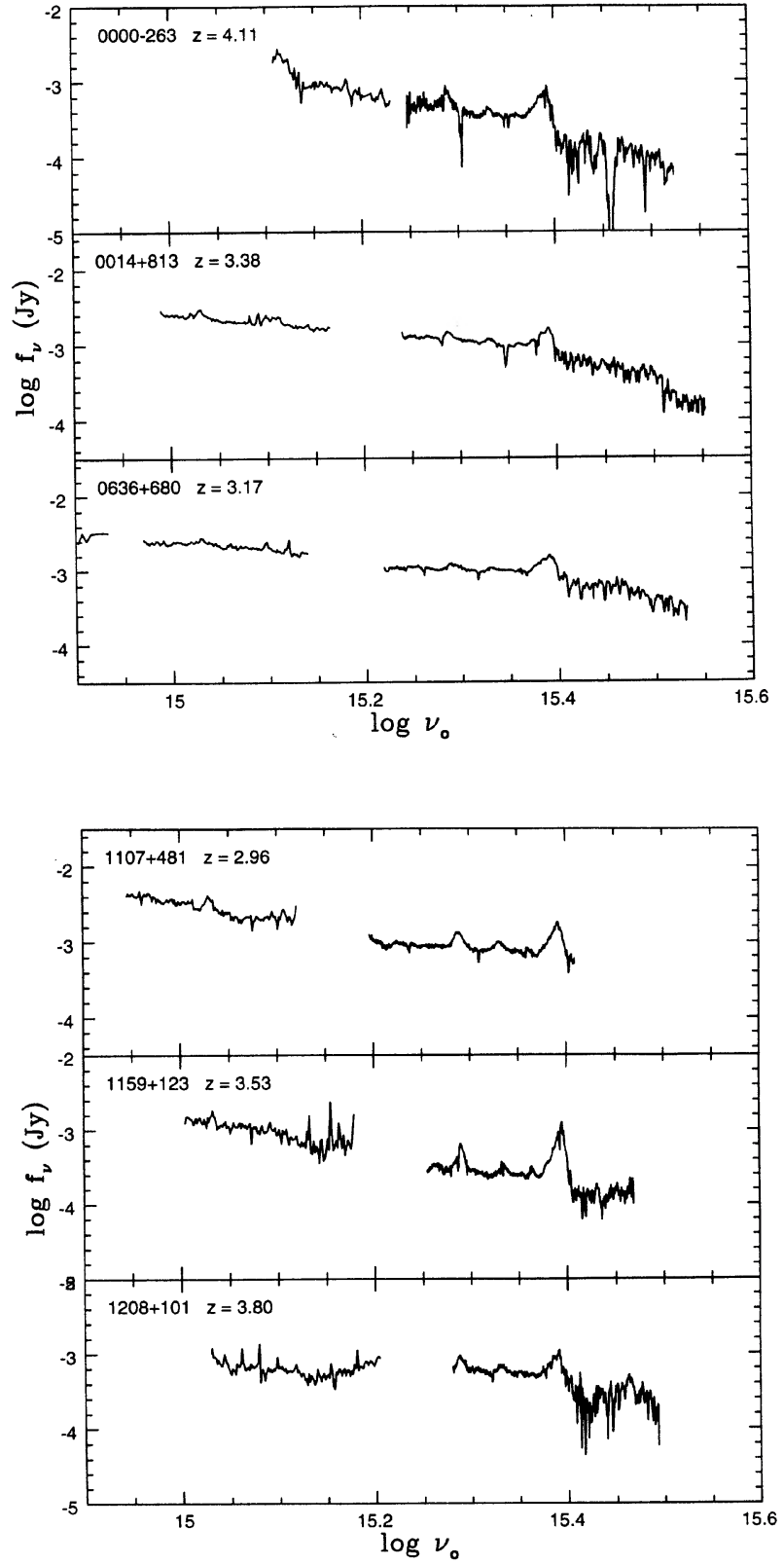

FIG. 7. Spectrophotometry of six high-redshift quasars obtained with the Germanium Spectrometer at the MMT and B\&C Spectrograph at the Steward Observatory 90 in. Logarithm of the flux in Janskies vs logarithm of rest frequency in $\mathrm{Hz}$.

fluctuations could be neglected. As a result, the spectral resolution is low, and the blended Lyman $\alpha$ forest depresses the observed continuum shortward of Lyman $\alpha$. Spectra of the Lyman $\alpha$ forest with high enough resolution to detect the continuum between individual lines can only be obtained with narrow slits, and are therefore difficult to flux calibrate. However, the two types of data can be combined to determine the unabsorbed continuum (Kuhn 1994). For 0014 +813 , we have used high resolution spectra of the Lyman $\alpha$ forest presented in Bechtold (1994) to correct the spectrophotometry for this "line blanketing." The result is shown in the solid line "c" next to spectrum "b" in Fig. 9.

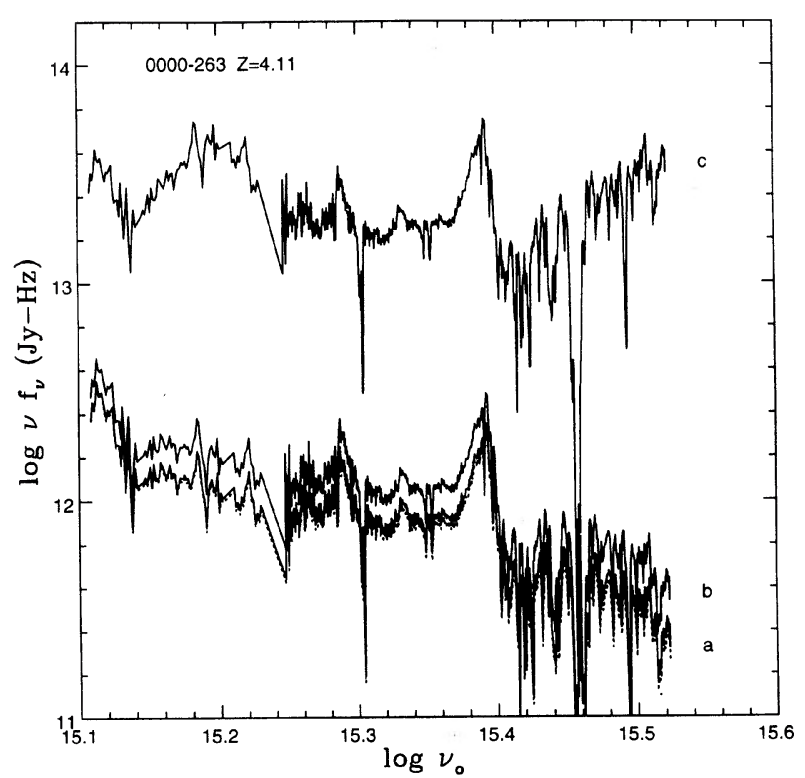

FIG. 8. Near-IR and optical spectral energy distribution of 0000-263 with different reddening corrections. (a) Corrected for galactic extinction, with $\mathrm{E}(B-V)=0.033$ at $z=0$. (b) Corrected for galactic extinction and extinction at $z=3.39$ with $\mathrm{E}(B-V)=0.042$, corresponding to a dust-to-gas ratio for the intervening absorber which is $1 / 10$ the Milky Way value. (c) Corrected for galactic extinction and extinction at $z=3.39$ with $\mathrm{E}(B-V)=0.42$, corresponding to a dust-to-gas ratio for the intervening absorber which is equal to the Milky Way value. In all cases, the Milky Way reddening law was assumed. The dotted line is the observed spectrum, before any correction for extinction.

For 0000-263 dust associated with an intervening damped Lyman $\alpha$ system at $z_{\text {abs }}=3.39$ may redden the spectrum significantly. Obviously, the dust-to-gas ratio and reddening law of this galaxy at $z=3.39$ is not known. Pei et al.

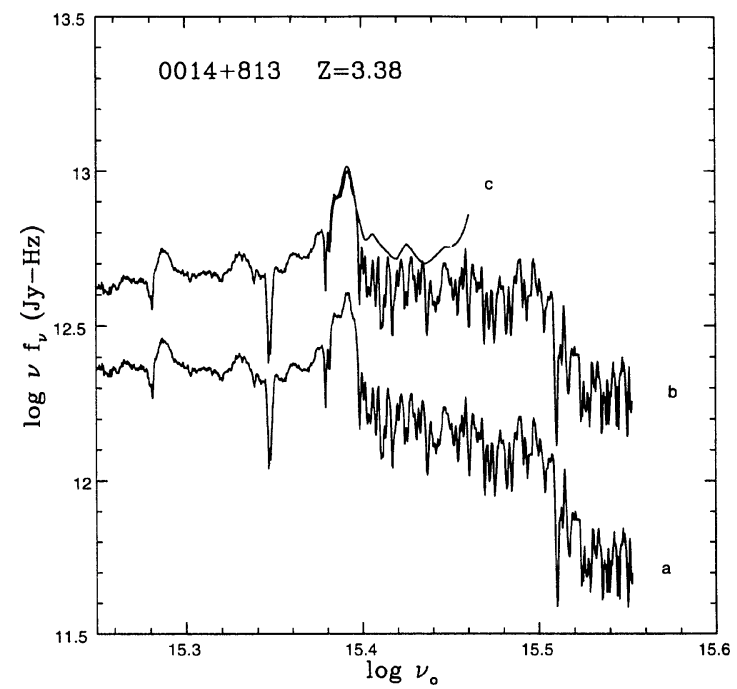

FIG. 9. Near-IR and optical spectral energy distribution of $0014+813$. (a) Observed. (b) Corrected for galactic extinction, with $\mathrm{E}(B-V)=0.30$. (c) Corrected for galactic extinction, and Ly $\alpha$ forest absorption; see text. 
(1991) found that the dust-to-gas ratio in a sample of $z=2$ damped Ly $\alpha$ systems is probably $\sim 1 / 10$ that of the Milky Way; recently, Ge \& Bechtold (1994) found that the dust-togas ratio may be even lower at higher $z$. In any event, in Fig. 8 we show what the SED looks like if we derreden it assuming the Milky Way reddening law and a dust-to-gas ratio 1/10 the Milky Way value (spectrum b) and a dust-to-gas ratio equal to the Milky Way value (spectrum c). For spectrum $\mathrm{c}$, the reddening correction seems to be too large: the spectrum rises in the UV, and shows a large bump produced by the $2200 \AA$ feature in the reddening law. The SMC reddening law may be more appropriate if the metallicity is low for this high redshift galaxy (cf. Pettini et al. 1993), in which case there is no $2200 \AA$ feature. Clearly, since we don't know the detailed shape of the reddening law, the shape of the intrinsic SED is uncertain. However, it is plausible that the true unreddened spectrum lies somewhere between $b$ and $c$; therefore, we use both in the analysis in Sec. 7.

Damped Ly $\alpha$ systems are also known in 1946-7658 (which was corrected for reddening in Kuhn et al. 1994) and 0420-388 (see discussion in Paper I). PKS 2000-333 has a high column density absorber which is probably damped (Saucedo \& Bechtold 1994). However, in the discussion below, specifically when computing the optical luminosities of the quasars, we neglect any correction for intervening dust, since these corrections are negligible if the dust-to-gas ratio in these objects is $1 / 10$ the Milky Way value. For any particular object, however, there is the possibility that there are undetected intermediate redshift absorbers with sufficient column to cause substantial reddening.

In $0014+813$, there are no damped Ly $\alpha$ absorbers known along the line of sight (Rauch et al. 1991; Bechtold 1994) and the known metal-line absorption systems probably have low enough $N_{\mathrm{H} \text { I }}$ that they cause little or no reddening even if their dust-to-gas ratio is equal to the Milky Way value.

\section{FAR-INFRARED IRAS PHOTOMETRY}

IRAS ADDSCAN processing was carried out at IPAC for all objects in the sample except $0438-436,2000-330$, and 2126-158, which were the subjects of deep pointed observations by Neugebauer et al. (1986). The resulting flux densities and upper limits are summarized in Table 9. For 0014 $+811,0207-398,1107+484$, and $1208+101$, the ADDSCAN median processing reported a net signal in one IRAS band, but examination of the individual scans suggested that these detections are noise related and therefore not real.

In the case of $0420-388$, a real source is apparently detected at $60 \mu \mathrm{m}$, with a flux of $0.150 \pm 0.034 \mathrm{Jy}$. However, the IRAS source is about $1^{\prime}$ south of the optical position. Since there is nothing visible on the Palomar Sky Survey at the IRAS source position, and $1^{\prime}$ is approximately the $90 \%$ error radius for source positions, we tentatively identify the IRAS source with the quasar.

\section{NOTES ON INDIVIDUAL OBJECTS}

Figure 10 shows the spectral energy distributions (SEDs) from the $x$-rays to radio for the six radio-loud quasars in the
TABLE 9. IRAS results.

\begin{tabular}{lccccc}
\hline \hline Quasar & $z_{\text {em }}$ & F12 & F25 & F60 & F100 \\
\hline $0000-262$ & 4.11 & $<0.11$ & $<0.12$ & $<0.11$ & $<0.35$ \\
$0014+811$ & 3.38 & $<0.05$ & $<0.07$ & $<0.12$ & $<0.35$ \\
$0055-262$ & 3.65 & $<0.09$ & $<0.09$ & $<0.08$ & $<0.47$ \\
$0130-402$ & 3.03 & $<0.08$ & $<0.08$ & $<0.14$ & $<0.50$ \\
$0207-398$ & 2.81 & $<0.10$ & $<0.08$ & $<0.72$ & $<0.24$ \\
$0420-388$ & 3.12 & $<0.05$ & $<0.07$ & $0.15 \pm 0.03$ & $<0.30$ \\
$0438-436$ & 2.85 & $0.025 \pm .008$ & $<0.03$ & $0.12 \pm .01$ & $0.279 \pm .047$ \\
$0636+681$ & 3.17 & $<0.08$ & $<0.07$ & $<0.10$ & $<0.47$ \\
$1107+484$ & 2.99 & $<0.07$ & $<0.08$ & $<0.11$ & $<0.24$ \\
$1159+123$ & 3.53 & $<0.09$ & $<0.12$ & $<0.10$ & $<0.27$ \\
$1208+101$ & 3.80 & $<0.14$ & $<0.16$ & $<0.12$ & $<0.28$ \\
$1946+768$ & 3.02 & $<0.11$ & $<0.08$ & $<0.12$ & $<0.38$ \\
$2000-330$ & 3.78 & $<0.02$ & $<0.04$ & $<0.03$ & $<0.08$ \\
$2126-158$ & 3.28 & $<0.04$ & $<0.08$ & $0.08 \pm 0.02$ & $<0.19$ \\
\hline
\end{tabular}

Notes to TABLE 9

All entries were derived from ADDSCANS, except for 0438-436, 2000-330, 2126-158, which were the subjects of deep pointed observations by Neugebauer et al. (1986). All fluxes in Jy. Upper limits are $3 \sigma$.

sample. Figure 11 shows the SEDs for the radio-quiet quasars. In this section we discuss details of the SEDs for individual objects.

$$
5.10000-263, z_{e m}=4.11
$$

This exceptional quasar was discovered in an optical search by Hazard (Webb et al. 1988), and its absorption line spectrum has been studied extensively (Sargent et al. 1989; Steidel 1990; Turnshek et al. 1991; Webb et al. 1992; Bechtold 1994). An optically thick Lyman limit system associated with a damped Ly $\alpha$ line at $z=3.392$ (Webb et al.1988; Steidel \& Hamilton 1992) cuts off the quasar continuum below $4000 \AA$. VLA data indicate that this quasar is radio quiet, with a $3 \sigma$ upper limit to the $6 \mathrm{~cm}$ flux of $0.39 \mathrm{mJy}$ (Kuhn 1994). Schneider et al. (1989) and Sargent et al. (1989) obtained optical spectrophotometry in September 1988 and October 1987, respectively, and in both cases the continuum level is consistent with our data obtained in November 1989. Thus, the quasar has not varied perceptibly during this period; unfortunately, the ROSAT data were obtained two years later, in December 1991.

We note that the coordinates of the quasar are $\alpha(1950.0)$ $=00 \mathrm{~h} 00 \mathrm{~m} 49.5 \mathrm{~s}, \delta(1950.0)=-26^{\circ} 20^{\prime} 01^{\prime \prime}$, as measured by Schneider et al. (1989). In the SIMBAD database, the truncated 1950.0 coordinates from the original Webb et al. (1988) paper are given $\left(\alpha=00 \mathrm{~h} 00 \mathrm{~m} 00 \mathrm{~s} \delta=-26^{\circ} 19^{\prime} 00^{\prime \prime}\right)$, and when these are precessed to J2000 coordinates, SIMBAD then gives a position with the usual significant figures, which happens to fall very close to the brightest source in the field (with 375 counts in 0.1 to $2.4 \mathrm{keV}$ ). The proper identification for the quasar is a much weaker source about an arcminute away. In Fig. 11, we plot the $\mathrm{x}$-ray spectral fit \#2 of Table 6, where the $\mathrm{x}$-ray absorption is fixed at the galactic value. The $J H K$ photometry is from Kuhn (1994).

$$
5.20014+813, z_{e m}=3.38
$$

$0014+813$ is a very luminous, flat-spectrum quasar discovered by Kuhr et al. (1983). The radio spectrum is given by Kuhr et al. (1986). High resolution VLA and VLBI maps 

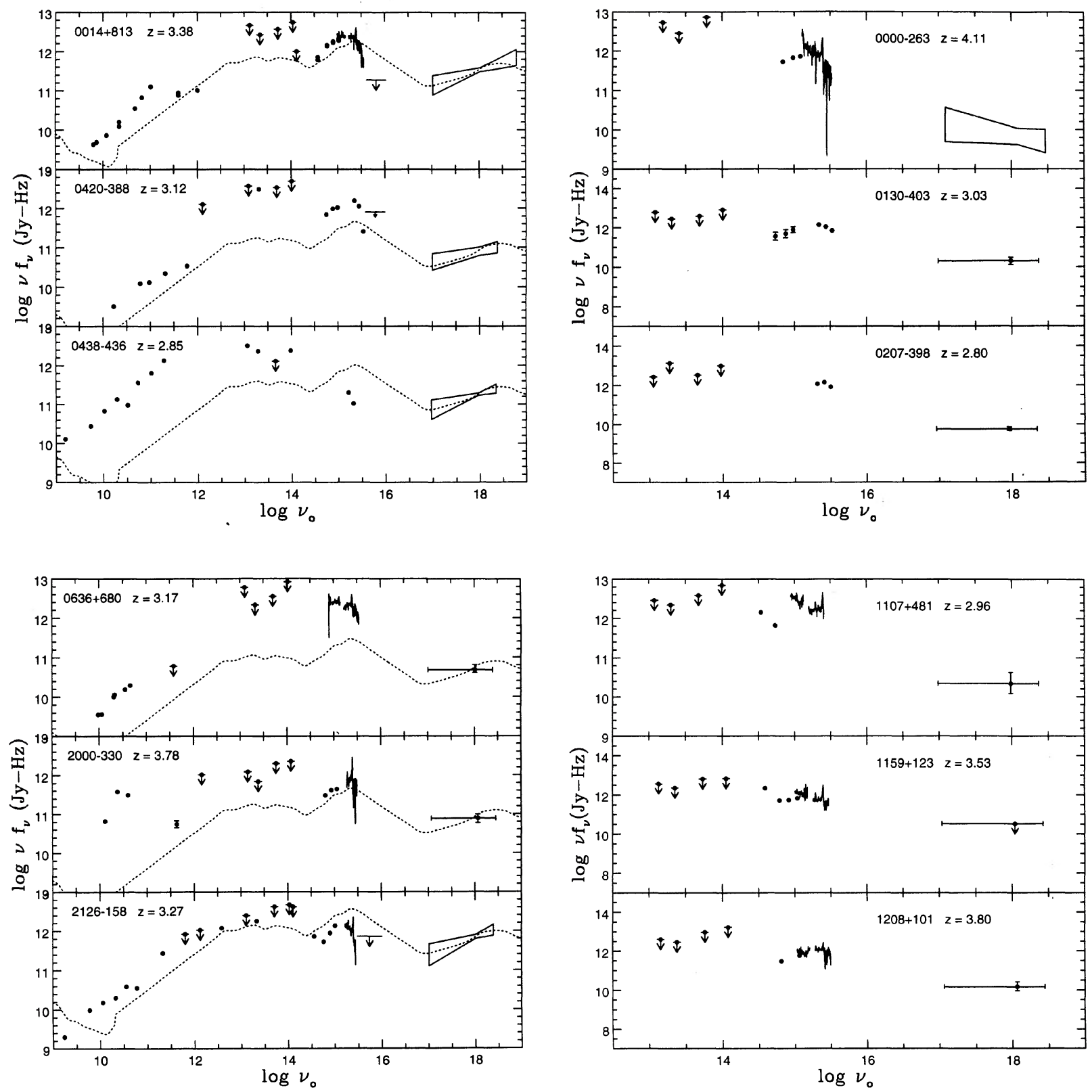

FIG. 10. Spectral energy distributions for six high-redshift radio-loud quasars (see text for references). Dotted line is the mean spectral energy distribution for low-redshift, radio-loud quasars from the compilation of Elvis et al. (1994b), with arbitrary normalization to the $1 \mathrm{keV}$ flux.

show a faint jet-like extension 0.6 arcsec to the south-east (Kuhr et al. 1986). 0014+813 was detected in the mm region by Steppe et al. (1988).

Kuhr et al. (1984) observed $0014+813$ in the near infrared in 1982-1984, and give $J H K$ magnitudes, and narrow band fluxes in the $2 \mu \mathrm{m}$ region, including $\mathrm{H} \beta$ and [O III] $\lambda 5007$, obtained with the CVF system at the MMT. These measurements lie below the level expected from the GeSpec spectrophotometry presented here, which suggested a small amount of variability. However, Kuhn (1994) observed 0014 +813 in 1992 and 1993, and found no evidence for variability at $J, H, K$, or $L$, compared to the earlier data. She also

FIG. 11. Spectral energy distributions for eight high-redshift radio-quiet quasars (see text for references).

placed an upper limit on the $10 \mu \mathrm{m}$ flux with the MMT bolometer; this photometry is also shown in Fig. 10.

An optically thick Lyman limit cuts off the spectrum below $\sim 3500 \AA$ in the optical, but the corresponding Ly $\alpha$ line is not damped (Sargent et al. 1989). 0014+813 was observed in the UV with IUE and not detected (e.g., Lanzetta et al. 1993). 0014+813 was also observed with the FOS on $H S T$, and the archived spectrum reveals that it was not detected to a more sensitive limit than the IUE data yield, with $f_{\lambda}<0.3 \times 10^{-15} \mathrm{erg} \mathrm{s}^{-2} \mathrm{~cm}^{-2} \AA^{-1}$ between 1250 and $1800 \AA$. We plot the $H S T$ limit in Fig. 10.

Finally, we note that $0014+813$ may be identified with a HEAO-A1 source (Bierman et al. 1992). The composite fit to 
the EXOSAT ME and PSPC data, given in Table 6 (fit 6), is plotted in Fig. 10. The rest of the spectral energy distribution is drawn from Kuhr et al. (1986), Steppe et al. (1988), and Kuhn (1994).

$$
5.30055-264, z_{e m}=3.67
$$

This object was discovered in an optical survey by Hazard \& McMahon (1985). Unfortunately, the positions in that paper and Hewitt \& Burbidge (1987) are incorrect by 30 arcmin in declination, and we mistakenly pointed the PSPC at those coordinates. The correct coordinates are $\alpha(1950)=00 \mathrm{~h}$ $55 \mathrm{~m} \mathrm{32.6s}$, and $\delta(1950)=-26^{\circ} 59^{\prime} 25^{\prime \prime}$ (Schneider et al. 1992a); thus, the quasar is well away from the center of the PSPC field, and in fact is under the support ribs. We have therefore eliminated this object from the sample.

$$
5.40130-403, z_{\text {em }}=3.03
$$

Osmer \& Smith (1976) discovered this object on an objective-prism plate and obtained spectrophotometry on 1976 Jan 2-3. The continuum level at $1475 \AA$ rest was $\log f_{\nu}=-3.42 \mathrm{Jy}$. Adam (1985) obtained $U B V$ photometry three times between 1977 and 1980, and Rodriquez-Espinosa et al. (1988) obtained $J H K$ photometry in July 1986. The continuum appears to be fainter in the Osmer \& Smith (1976) determination than in Adam's broadband colors, perhaps due to contamination from the bright Ly $\alpha$ line in Adam's $V$ magnitude. The spectral energy distribution shown in Fig. 11 is drawn from Adam (1985) and RodriquezEspinosa et al. (1988).

$$
5.50207-398, z_{e m}=2.81
$$

This quasar was discovered in the Tololo Curtis-Schmidt Survey by Whelan et al. (1979) and has been the subject of several absorption line studies. Adam (1985) gives $U, B$, and $V$ magnitudes, which are plotted in Fig. 11. Zamorani et al. (1981) detected 0207-398 with the Einstein IPC. The reprocessed IPC data listed in Wilkes et al. (1994) give a count rate of $48.66 \pm 11.98$ counts in the 0.2 to $3.5 \mathrm{keV}$ band in $5931.5 \mathrm{~s}$ on 1979 June 16 . Assuming an $\mathrm{x}$-ray spectral index of $\alpha_{E}=0.5$, and the galactic absorption column of $N_{\mathrm{H} I}$ $=1.53 \times 10^{20} \mathrm{~cm}^{-2}$, this corresponds to a monochromatic luminosity at $2 \mathrm{keV}$ of $2.6 \pm 0.6 \times 10^{28} \mathrm{erg} \mathrm{s}^{-1} \mathrm{~Hz}^{-1}$ for $H_{0}=50 \mathrm{~km} \mathrm{~s}^{-1} \mathrm{Mpc}^{-1}$ and $q_{0}=0$. With the PSPC we saw $62 \pm 13$ counts which implies a monochromatic luminosity at $2 \mathrm{keV}$ of $l_{x}=4.8 \pm 0.9 \times 10^{27} \mathrm{erg} \mathrm{s}^{-1} \mathrm{~Hz}^{-1}$ with the same assumptions. These are different at the $3.2 \sigma$ level, indicating a marginally significant fading in the $\mathrm{x}$-rays of $0207-398$ on an observed time scale of $\sim 12 \mathrm{yr}$, or $\sim 3 \mathrm{yr}$ in the rest frame.

$$
5.60420-388, z_{\text {em }}=3.12
$$

The PSPC spectrum for this quasar was discussed in $\mathrm{Pa}$ per I. $0420-388$ was discovered on a Schmidt plate by Osmer \& Smith (1980), and turns out to be a radio loud quasar, with perhaps a Gigahertz Peaked spectrum (O'Dea et al. 1991). In Fig. 10 we plot infrared $J H K$ colors from Hyland \& Allen (1982) and optical colors from Adam (1985). The radio data is from Condon et al. (1981) and
Robson et al. (1985). An optically thick Lyman limit system, associated with a damped $\operatorname{Ly} \alpha$ line at $z_{\mathrm{abs}}=3.08$, cuts off the spectrum below $3750 \AA$ (Atwood et al. 1985). Lanzetta et al. (1993) show the archival IUE spectrum, which shows no flux in the EUV. We show their upper limit $\left(f_{\lambda}<0.2 \times 10^{-14}\right.$ erg $\mathrm{s}^{-1} \mathrm{~cm}^{-2} \AA^{-1}$ ) in Fig. 10.

We note that the IRAS $60 \mu \mathrm{m}$ detection for this object, if confirmed, would imply an extraordinary "IR excess" for this quasar. It would be one of the most luminous objects in the Universe, with the majority of the energy being emitted in the far IR (cf. Cutri et al. 1994).

$$
5.70438-436, z_{\text {em }}=2.85
$$

This quasar is exceptionally luminous in the radio (Morton et al. 1978) but faint in the optical $(V=19.8$, Impey \& Tapia 1990). Thus, very little detailed optical or near IR spectroscopy has been done. However, it was detected in deep IRAS observations at 12, 60, and $100 \mu \mathrm{m}$ (Neugebauer et al. 1986), which is unusual for such a high redshift object. The optical light is polarized (Impey \& Tapia 1990), and the VLBI map shows a "core-jet" morphology, with two components separated by 35 milliarcsec (Preston et al. 1989). Thus, this object probably differs from the others in the sample in that the nonthermal component at all wavelengths is important. Its x-ray spectrum shows soft absorption in excess of the galactic H I column density (Wilkes et al. 1992; Paper I).

The spectral energy distribution shown in Fig. 10 is drawn from Wall \& Peacock (1985), Neugebauer et al. (1986), Impey \& Tapia (1990), Wright et al. (1991), Fugmann \& Meisenheimer (1988), and Quiniento et al. (1988).

$$
5.80636+680, z_{\text {em }}=3.17
$$

Kuhr (1977) identified this quasar as the optical counterpart of a radio source from the $5 \mathrm{GHz}$ survey of Pauliny-Toth et al. (1978), and estimated its magnitude as $V=19$ from the Palomar Sky Survey. It appears to be substantially brighter (see also Sargent et al. 1989), both on the Palomar survey, and in our data $(V \sim 17.5)$. It is a candidate "GHz Peaked" radio source (O'Dea et al. 1991), which is unresolved with the VLA at $8.4 \mathrm{GHz}$ (Patnaik et al. 1992) and VLBI (Preston et al. 1985). The radio and submillimeter data plotted in Fig. 10 are from Preston et al. (1985), Steppe et al. (1988), Becker et al. (1991), Gregory \& Condon (1991), White \& Becker (1992), and Patnaik et al. (1992). An optically thick Lyman limit system at $z_{\text {abs }}=2.90$ cuts off the continuum below $\sim 3600 \AA$ (Sargent et al. 1989).

The PSPC spectrum of $0636+680$ was discussed in Paper I. Although only 68 net photons were detected, spectral fits with the power law spectral index fixed at $\alpha_{E}=0.7$ implied the presence of absorption in excess of the galactic $N(\mathrm{H} \mathrm{I})$, with a column similar to the absorption seen in $0438-436$ and 2126-158. The hardness ratio is consistent with these conclusions also. 


$$
5.91107+481, z_{\text {em }}=2.96
$$

Sanduleak \& Pesch (1989) suggested that this object is a bright, high redshift quasar, and the slit spectrum shown in Fig. 7 confirms that it is a quasar at $z_{\mathrm{em}}=2.958$. It happened to be included in the field of a deep radio survey at 4850 MHz by Maslowski et al. (1984), and was not detected. The upper limit of $17 \mathrm{mJy}$ implies that this quasar is radio quiet. Kuhn (1994) detected $1107+481$ at $6 \mathrm{~cm}$ with the VLA, and again it appears to be radio quiet. $K$ and $L$ photometry obtained in 1992 by Kuhn (1994) does not agree in absolute level with the optical and GeSpec data obtained in 1989. Optical or $J$ photometry will be necessary to verify whether this is real variation, or a problem with the calibration of the spectrophotometry.

$$
5.101159+123, z_{\text {em }}=3.53
$$

Hazard et al. (1984) found this quasar on an objective prism plate. Condon et al. (1982) conducted a deep VLA survey at $1411 \mathrm{MHz}$ which included this quasar, and did not detect it, with an upper limit of 5 mJy. Kuhn (1994) also placed an upper limit with the VLA at 6 and $20 \mathrm{~cm}$. Thus, $\mathrm{Q} 1159+123$ is clearly radio quiet. An optically thick Lyman limit system at $z_{\mathrm{abs}} \approx 3.5$ cuts off the continuum below $\sim 4150 \AA$ (Antonucci 1989). Unfortunately, this object was scheduled for only $1800 \mathrm{~s}$ of integration with the PSPC, and was not detected. JHKL photometry by Kuhn (1994) is plotted in Fig. 11.

$$
5.111208+101, z_{e m}=3.80
$$

This quasar was discovered in an objective prism survey by Hazard et al. (1986). Condon et al.'s (1982) deep VLA survey at $1411 \mathrm{MHz}$ included this quasar, and did not detect it with an upper limit of $4 \mathrm{mJy}$. Kuhn (1994) also placed upper limits at 6 and $20 \mathrm{~cm}$ with the VLA. Thus, Q1208 +101 is clearly radio quiet. In the optical spectrum of Sargent et al. (1986), it appears that there is no optically thick Lyman limit in the optical range, and the continuum is detected to the rest wavelength of $\sim 670 \AA$ in the quasar frame.

Recently, it has been discovered that Q1208+101 is gravitationally lensed (Bahcall et al. 1992a; Magain et al. 1992; Bahcall et al. 1992b). The two detected images are separated by 0.47 arcsec. Given the large apertures with which all the data shown in Fig. 11 were obtained, and the spatial resolution of the PSPC, the spectral energy distribution is the sum of both images. Although the lens itself has not been identified, given the separation, it is probably an intervening galaxy (Bahcall et al. 1992b). The factor by which the continuum is magnified is not known. However, a weak constraint is given by Bechtold (1994) who noted that the Lyman $\alpha$ forest in this object does not show a particularly weak "proximity effect" for its apparent magnitude, as might be expected if the intrinsic continuum is very much fainter than observed.

$$
5.121946+768, z_{e m}=3.02
$$

Kuhn et al. (1994) discuss the spectral energy distribution of this quasar in detail.

$$
5.132000-330, z_{e m}=3.78
$$

Discovered in the course of the Parkes $2.7 \mathrm{GHz}$ flat spectrum survey, this object clearly has a " $\mathrm{GHz}$ peaked" radio spectrum (Peterson et al. 1982; Savage et al. 1990; O'Dea et al. 1991), although most of the radio data are unpublished. The radio source is unresolved at VLBI resolution $(<50$ milliarcsec, full width half maximum, Preston et al. 1989), and unpolarized at $6 \mathrm{~cm}$ (Saikia \& Shastri 1984). Radio and submillimeter fluxes from Wright et al. (1991), Quiniento \& Cerosimo (1993), and Steppe et al. (1988) are shown in Fig. 10. An upper limit at $962 \mu \mathrm{m}$ is given by Robson et al. (1985). PKS 2000-330 was the subject of a deep pointed observation with the IRAS satellite by Neugebauer et al. (1986), but was not detected in any waveband. Near IR photometry from Rodriquez-Espinosa et al. (1988) matches our optical spectrophotometry obtained more recently (Table 8 , Fig. 10) indicating that the quasar has not varied. An optically thick Lyman limit at $z_{\text {abs }}=3.548$ cuts off the continuum below $\sim 4200 \AA$ (Sargent et al. 1989, and references therein).

$$
5.142126-158, z_{e m}=3.27
$$

This quasar was discovered in the course of the Parkes radio survey (Condon et al. 1977; Jauncey et al. 1978) and has a candidate GigaHertz Peaked radio spectrum. It is unresolved at VLBI resolution (Wehrle et al. 1984), and unpolarized in the optical (Wills et al. 1992). Soifer et al. (1983) report $J H K$ colors, and Neugebauer et al. (1986) detected $2126-158$ at $60 \mu \mathrm{m}$ with IRAS. Radio data are given by Robson et al. (1985), Browne \& Perley (1986), Steppe et al. (1988), Wright et al. (1991), and Quiniento \& Cersoimo (1993). An optically thick Lyman limit system cuts off the spectrum below $\sim 3600 \AA$ (Sargent et al. 1989). IUE archival data presented by Lanzetta et al. (1993) show that 2126-158 was not detected, with an estimated upper limit of $f_{\lambda}<0.2 \times 10^{-14} \mathrm{erg} \mathrm{cm}^{-2} \mathrm{~s}^{-1} \AA^{-1}$. This is shown in Fig. 10.

Optical spectrophotometry of 2126-158 in 1978 by Oke \& Korycansky (1982), in 1987 by Sargent et al. (1989) and in 1989 by us, all agree in absolute level, indicating no variation. Kuhn (1994) measured JHKL and obtained an upper limit at $10 \mu \mathrm{m}$ in 1993. The recent $J$ and $H$ measurements agree with the Soifer et al. (1983) measurements which were obtained in 1981. The new $K$ measurement is $40 \%$ higher than Soifer et al.'s measurement which was taken in 1978. While it is possible that there has been some real variation at $K$ (which corresponds to $\sim 5500 \AA$ in the rest frame), this explanation seems unlikely, given the lack of variation at other wavelengths. The difference may result from changes in photometric calibrations or standard star networks between 1978 and 1993, although one would expect these to amount to at most $\sim 10 \%$. In any event, we adopt the Kuhn (1994) measurements in Fig. 10, since they were obtained nearer in time to the ROSAT data.

The $\mathrm{x}$-ray spectrum of this quasar from the PSPC is discussed in Paper I, and Sec. 2.5. In addition, 2126-158 was detected with the Einstein IPC (Zamorani et al. 1981). The PSPC spectrum is plotted in Fig. 10 (fit 1 of Table 6). 


\section{SPECTRAL ENERGY DISTRIBUTIONS}

Figure 10 shows the spectral energy distributions (SEDs) from the $\mathrm{x}$-rays to radio for the six radio-loud quasars in the sample. The mean SED in the sample of low redshift radioloud quasars given in Elvis et al. (1994b) is shown by the dotted line. This mean SED has been arbitrarily shifted to match the X-ray fluxes. Since the Elvis et al. (1994b) sample consisted of the objects with enough counts detected in the IPC to do meaningful spectral fits, it contains very $x$-ray bright objects compared to average optically selected quasars, and compared to the quasars in our sample. Thus, the mean SED, when normalized to the x-ray flux, falls below the observed points in the UV to radio wavebands. However, other than this systematic shift between the $\mathrm{x}$-rays and everything else, the high redshift objects seem very similar to the low redshift mean.

Variability is a concern. Since all quasars vary at some level, we had intended for the ground-based data to be obtained within a few weeks or months of the $\mathrm{x}$-ray data; this turned out to be infeasible. The ROSAT observations were delayed, and the first runs we had at the MMT and $2.3 \mathrm{~m}$ were clear, resulting in gaps of 1-2 yr between the groundbased and $\mathrm{x}$-ray observations. In the quasar rest frame this corresponds to $\sim 4-6$ months; many low-redshift AGN are seen to vary on these time scales. However, as we discussed for individual objects above, few of them varied in the 3-5 yr before the ROSAT observations. Also, we have obtained optical or IR photometry for several of the objects after the ROSAT observations were done, and found no significant variation, in comparison with the data taken before the ROSAT observations. Three objects had been detected by previous X-ray satellites, and of these one (0207-398, see Sec. 5.5), possibly two (2126-158, see Sec. 2.5$)$ have varied significantly in the $\mathrm{x}$-rays. However, all in all, we do not think that variability is a significant source of uncertainty in discussing the spectral energy distributions of the objects.

$$
6.1 \alpha_{o x} \text { and } \alpha_{x r}
$$

Here we discuss the spectral energy distributions of our program objects based on ratios of the flux in broadly defined optical/IR, X-ray and radio bands. In Sec. 7, we discuss the detailed shape of the SED's.

The correlations between optical, x-ray and radio luminosities for low redshift quasars have been discussed extensively, based on samples of quasars observed with the Einstein IPC (Zamorani et al. 1981; Avni \& Tananbaum 1982, 1986; Kriss \& Canizares 1985; Worrall et al. 1987). For optically selected, and presumably mostly radio quiet quasars, the ratio of $x$-ray to optical flux decreases with increasing optical luminosity, and is only weakly dependent on redshift. Radio-loud quasars were found to have a larger $\mathrm{x}$-ray to optical flux ratio compared to radio-quiet objects of the same optical luminosity, implying that an extra component to the $\mathrm{x}$-ray emission is present, associated with the radio emission. No significant dependence on redshift is seen for this effect. Since our sample extends the range of redshift and luminosity observed, we reexamine these trends below.
TABLE 10. Radio, optical, and x-ray luminosities and $\alpha_{\mathrm{ox}}$.

\begin{tabular}{|c|c|c|c|c|c|c|c|}
\hline Quasar & $z_{\epsilon ! n}$ & $1_{r}^{n}$ & $l_{o}^{b}$ & $1_{x}^{c}$ & $\alpha_{o x}^{d}$ & Comments & \\
\hline $0000-263$ & 4.11 & $<32.72$ & 33.26 & $\begin{array}{l}28.352 \\
28.398 \\
28.438 \\
28.555\end{array}$ & $\begin{array}{l}1.884 \\
1.866 \\
1.851 \\
1.806\end{array}$ & , & $\because$ \\
\hline $0014+813$ & 3.38 & 35.87 & 33.17 & $\begin{array}{l}29.589 \\
29.669 \\
29.776 \\
29.698\end{array}$ & $\begin{array}{l}1.374 \\
1.344 \\
1.303 \\
1.333\end{array}$ & PSPC & \\
\hline 0130-403 & 3.03 & - & 32.79 & $\begin{array}{l}28.294 \\
28.327 \\
28.356 \\
28.490\end{array}$ & $\begin{array}{l}1.726 \\
1.713 \\
1.702 \\
1.651\end{array}$ & & \\
\hline $0207-398$ & 2.81 & - & 32.5 & $\begin{array}{c}27.679 \\
27.694 \\
27.696 \\
27.925 \\
28.4\end{array}$ & $\begin{array}{c}1.850 \\
1.845 \\
1.844 \\
1.756 \\
1.57\end{array}$ & PSPC & \\
\hline $0420-388$ & 3.12 & 35.14 & 32.84 & $\begin{array}{c}28.889 \\
28.917 \\
28.939 \\
29.100 \\
28.86\end{array}$ & $\begin{array}{l}1.516 \\
1.506 \\
1.497 \\
1.435 \\
1.528\end{array}$ & PSPC & \\
\hline $0438+436$ & 2.85 & 36.50 & 32.18 & $\begin{array}{c}28.951 \\
28.966 \\
28.970 \\
29.196 \\
28.97\end{array}$ & $\begin{array}{l}1.239 \\
1.234 \\
1.232 \\
1.145 \\
1.232\end{array}$ & PSPC & \\
\hline $0636+680$ & 3.17 & 35.04 & 33.11 & $\begin{array}{l}28.675 \\
28.736 \\
28.814 \\
28.800\end{array}$ & $\begin{array}{l}1.704 \\
1.679 \\
1.649 \\
1.654\end{array}$ & & \\
\hline $1107+481$ & 2.96 & 32.45 & 33.00 & $\begin{array}{l}28.270 \\
28.287 \\
28.291 \\
28.519\end{array}$ & $\begin{array}{l}1.816 \\
1.809 \\
1.803 \\
1.720\end{array}$ & & \\
\hline $1159+123$ & 3.53 & $<32.58$ & 32.94 & $\begin{array}{l}<28.500 \\
<28.542 \\
<28.582 \\
<28.695\end{array}$ & $\begin{array}{l}>1.704 \\
>1.688 \\
>1.673 \\
>1.630\end{array}$ & & \\
\hline $1208+101$ & 3.82 & $<32.82$ & 33.11 & $\begin{array}{l}28.334 \\
28.373 \\
28.410 \\
28.540\end{array}$ & $\begin{array}{l}1.833 \\
1.818 \\
1.803 \\
1.754\end{array}$ & & \\
\hline $1946+768$ & 3.02 & 32.74 & 33.47 & $\begin{array}{l}28.372 \\
28.435 \\
28.519 \\
28.488\end{array}$ & $\begin{array}{l}1.957 \\
1.933 \\
1.900 \\
1.912\end{array}$ & & \\
\hline 2000-330 & 3.78 & 36.83 & 32.64 & $\begin{array}{l}29.046 \\
29.125 \\
29.229 \\
29.162\end{array}$ & $\begin{array}{l}1.379 \\
1.349 \\
1.309 \\
1.334\end{array}$ & & \\
\hline 2126-158 & 3.27 & 36.08 & 32.85 & $\begin{array}{c}29.814 \\
29.875 \\
29.949 \\
29.948 \\
29.77\end{array}$ & $\begin{array}{l}1.165 \\
1.142 \\
1.113 \\
1.113 \\
1.182\end{array}$ & PSPC & \\
\hline
\end{tabular}

Notes to TABLE 10

${ }^{\text {a}}$ Monochromatic luminosity at $5 \mathrm{GHz}$ (rest frame), in $\mathrm{erg} \mathrm{s}^{-1} \mathrm{~Hz}^{-1}$, for $H_{0}=50 \mathrm{~km} \mathrm{~s}^{-1} \mathrm{Mpc}^{-1}$ and $q_{0}=0.5$. For objects not detected, the $3 \sigma$ upper limit is giver. When no spectral information was available, $l_{\mathrm{r}}$ was computed from the ubserved $6 \mathrm{~cm}$ flux, with no $K$ correction. ${ }^{b}$ Monochromatic luminosity at $2500 \AA$ in the rest frame, as in (a). ${ }^{\mathrm{c}}$ Monochromatic luminosity at $2 \mathrm{keV}$ in the rest frame. See text. First, second, third, and fourth lines are for Models $1,2,3$, and 4 of Table 3, respectively. ${ }^{d} \alpha_{\text {ox }}$, see Eq. (5) in text.

In Table 10, we list $l_{\text {opt }}, l_{\mathrm{x}}$, and $\alpha_{\mathrm{ox}}$ for the program objects. $l_{\text {opt }}$ is the monochromatic luminosity at rest wavelength $2500 \AA, l_{x}$ is the luminosity at an energy of $2 \mathrm{keV}$ in the rest frame, and $l_{\mathrm{r}}$ is the luminosity in the radio, at $5 \mathrm{GHz}$. Note that we have measured the flux at $2500 \AA$ directly from the GESPEC data at an observed wavelength of $\sim 1 \mu \mathrm{m}$.

We assume $H_{0}=50 \mathrm{~km} \mathrm{~s}^{-1} \mathrm{Mpc}^{-1}$ and $q_{0}=0$ in order to be consistent with the compilation of Einstein IPC results by Wilkes et al. (1994). Then the luminosity is related to the observed flux $f_{0}$ by

$$
f_{0}=2.33 \times 10^{-62} l_{0} H_{0}^{2}(1+z) /\left[(1+z)-(1+z)^{1 / 2}\right]^{2},
$$




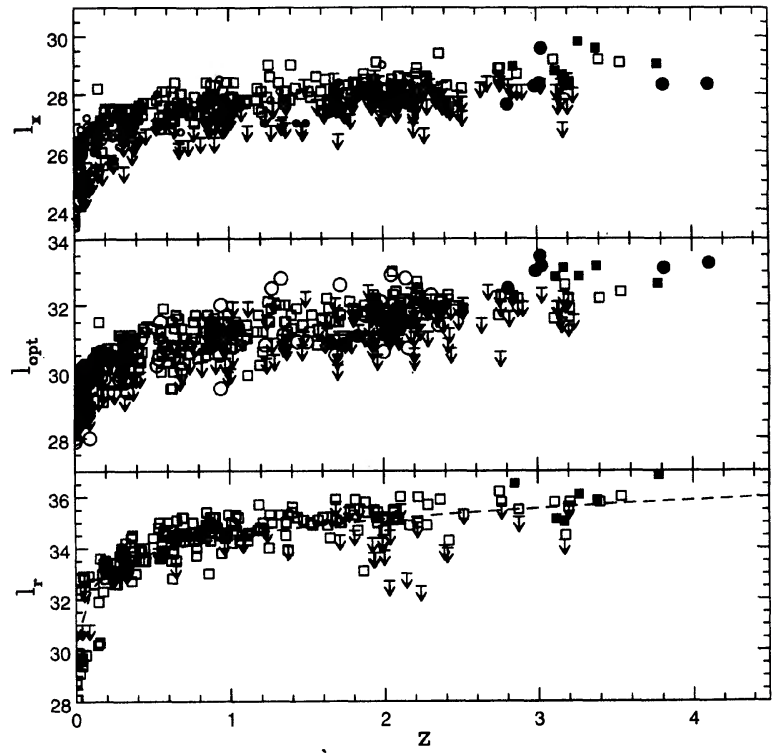

Fig. 12. Monochromatic luminosities in units of $\operatorname{erg~s}^{-1} \mathrm{~Hz}^{-1}$ at $2 \mathrm{keV}\left(l_{\mathrm{x}}\right)$, $2500 \AA\left(l_{\text {opt }}\right)$, and $5 \mathrm{GHz}\left(l_{\mathrm{r}}\right)$ as a function of redshift. $H_{0}=50$ $\mathrm{km} \mathrm{s}^{-1} \mathrm{Mpc}^{-1}$ and $q_{0}=0$ were assumed. Open squares: radio-loud quasars observed with the Einstein IPC. Open circles: radio-quiet quasars observed with the Einstein IPC. Filled squares: radio-loud quasars observed with the ROSAT PSPC. Filled circles: radio quiet quasars observed with the ROSAT PSPC. Dashed line indicates the luminosity as a function of redshift for (arbitrary) fixed observed flux.

where $f_{0}$ has units of $\mathrm{erg} \mathrm{cm}^{-1} \mathrm{~s}^{-1} \mathrm{~Hz}^{-1}$ and $l_{0}$ has units of $\operatorname{erg~s}^{-1} \mathrm{~Hz}^{-1}$. Following Zamorani et al. (1981), we then compute $\alpha_{\mathrm{ox}}$ where

$$
\alpha_{\text {ox }}=-\log \left(l_{\mathrm{x}} / l_{\text {opt }}\right) / \log \left(\nu_{\mathrm{x}} / \nu_{\text {opt }}\right),
$$

where $\log \nu_{\mathrm{x}}=17.6845$ for $2 \mathrm{keV}$ and $\log \nu_{\mathrm{opt}}=15.0791$ for $2500 \AA$; all quantities are in the rest frame of the quasar. The results for $\alpha_{\text {ox }}$ are listed in Table 10, for each of the four $\mathrm{x}$-ray spectral models described in Table 3. Note that the difference in $\alpha_{\mathrm{ox}}$ between assuming that the x-ray spectral index is $\alpha_{E}=0.5$ or 1.0 is less than $5 \%$.

For a comparison sample, we used the results from the Einstein Quasar and Seyfert 1 Galaxy Database by Wilkes et al. (1994) as obtained from the Einline on-line service. This database contains optical and $\mathrm{x}$-ray information for the 531 quasars and Seyfert 1 galaxies observed with the Einstein Observatory IPC from 1978 to 1981 . While the sample is quite heterogeneous, it is perhaps large enough to be representative. It includes several samples which are well observed, including the PG quasars. To investigate the radio properties of the sample, we cross-correlated this list with the the Veron-Cetty \& Veron (1989) and Hewitt \& Burbidge (1987) catalogs. The results are shown in Figs. 12 through 15.

First, in Fig. 12 we show the luminosity-redshift diagrams for the objects in the Einstein sample (open circles for radio quiet objects and open squares for radio loud), and the objects in the high redshift PSPC sample (filled circles for radio quiet objects and filled squares for radio loud). Objects which were not detected in the IPC are indicated by arrows
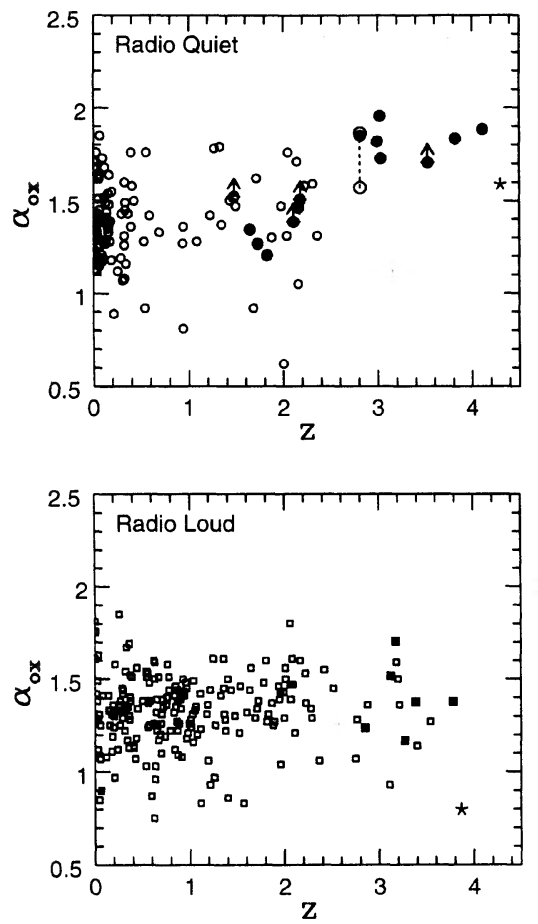

FIG. 13. $\alpha_{\mathrm{ox}}$ as a function of redshift for radio quiet and radio loud quasars. Open symbols are IPC results and closed symbols are ROSAT PSPC results presented here. The IPC and PSPC values for 0207-389 are connected by a dashed line. The $z=4.3$ quasar of Henry et al. (1994) and the $z=3.87$ quasar of Fink \& Briel (1993) are shown as five pointed stars.

in all panels. The dashed line shows the luminosity as a function of redshift for an object with constant observed flux (which is taken to be arbitrary). With the possible exception of the lowest redshift objects, the sample consists of objects of similar flux at all redshifts.

In Fig. 13, we plot $\alpha_{\text {ox }}$ as a function of redshift for the radio quiet and radio loud quasars. The open symbols are the Einstein data, and the filled symbols are the new PSPC objects. In order to be consistent with the Einstein results, we used the results for Model $1\left(\alpha_{E}=0.5\right.$, galactic absorption only) for the PSPC fluxes. Only the quasars detected with Einstein are shown in this plot; the upper limits are discussed below. The intermediate redshift objects from the 0130-403 field are shown, including the three which were not detected. Finally, we also plot two very high redshift quasars which have $x$-ray fluxes from the PSPC All-Sky Survey. Henry et al. (1994) discovered a $z=4.3$ radio-quiet quasar (Kollgaard et al. 1994), with $m_{A B}(1415 \AA)=19.3$ and $\alpha_{\text {ox }}=1.59$. Fink \& Briel (1993) used the PSPC to detect a previously known radio loud high redshift quasar, $1745+624$, which has $\alpha_{\text {ox }}=0.80$ at $z=3.87$ (Becker et al. 1992).

The high-redshift radio-quiet objects appear to be significantly more x-ray quiet (i.e., larger $\alpha_{\mathrm{ox}}$ ) than their low redshift counterparts. The seven objects with $z>2.5$ have a mean $\left\langle\alpha_{\text {ox }}\right\rangle=1.80 \pm 0.01$, compared to $\left\langle\alpha_{\text {ox }}\right\rangle=1.38 \pm 0.05$ for the 111 quasars detected in the IPC with $z<2.5$. From the Student's $T$-test, these means have a probability of being the same of only $1.0 \times 10^{-5}$. The range of $\alpha_{\text {ox }}$ seen in low red- 

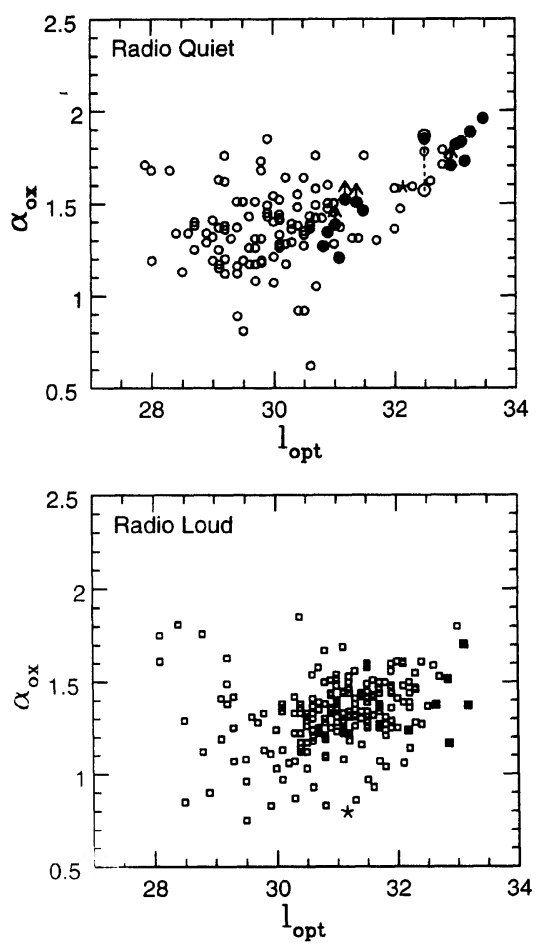

FIG. 14. $\alpha_{\mathrm{ox}}$ as a function of $l_{\mathrm{opt}}\left(\mathrm{erg} \mathrm{s}^{-1} \mathrm{~Hz}^{-1}\right)$ for radio loud and radio quiet quasars. Symbols are the same as Fig. 13.

shift radio-quiet quasars is 1.0-2.0 so that a change of $\alpha_{\mathrm{ox}}$ of 0.45 is large. It is also quite large in absolute terms, corresponding to a change in $L_{X} / L_{\text {opt }}$ of a factor 15 . However, the Kendall Tau test and the Spearman Rank-Order test show only a marginal correlation; the probability that there is a significant correlation is only about $94 \%$ or $1.9 \sigma$ for either statistic. Since both these tests are for correlations rather than discontinuities, this may suggest that the change in $\alpha_{\mathrm{ox}}$ is due to a jump, not a trend.

The high redshift radio quiet objects are also the most luminous optically, and the analysis of the IPC data has shown that $\alpha_{\text {ox }}$ increases with increasing $l_{\text {opt }}$ (Avni \& Tananbaum 1982, 1986; Kriss \& Canizares 1985; Worrall et al. 1987; Wilkes et al. 1994). If we extrapolate the dependence of $\alpha_{\mathrm{ox}}$ on $l_{\mathrm{opt}}$ for the radio quiet quasars given by Worrall et al. (1987), then for $\log l_{\mathrm{opt}}=33 \mathrm{erg} \mathrm{s}^{-1} \mathrm{~Hz}^{-1}$ (typical of the quasars in our sample), the expected $\alpha_{\text {ox }}=1.78$, which is consistent with the observed mean $\left\langle\alpha_{\text {ox }}\right\rangle \sim 1.8$. At the same $l_{\text {opt }}$, the radio loud, steep spectrum quasars are expected to have $\alpha_{\mathrm{ox}}=1.66$; the radio-loud, flat spectrum sources, $\alpha_{\text {ox }}=1.45$; and the radio-loud, steep-spectrum, but compact sources, $\alpha_{\mathrm{ox}} \approx 1.6$. However, the radio loud quasars at high redshift are more $\mathrm{x}$-ray loud than their counterparts at lower $z$. Instead, the radio loud objects appear to have nearly constant $\alpha_{\text {ox }}$ with redshift and $L_{\text {opt }}$, with a mean of $\sim 1.4$. Figure 14 shows $\alpha_{\text {ox }}$ vs $l_{\text {opt }}$ for the radio quiet and radio loud objects. Here there appears to be a trend of increasing $\alpha_{\mathrm{ox}}$ with increasing $l_{\mathrm{opt}}$ for the radio quiet objects with $\log l_{\mathrm{opt}}>32.0$,
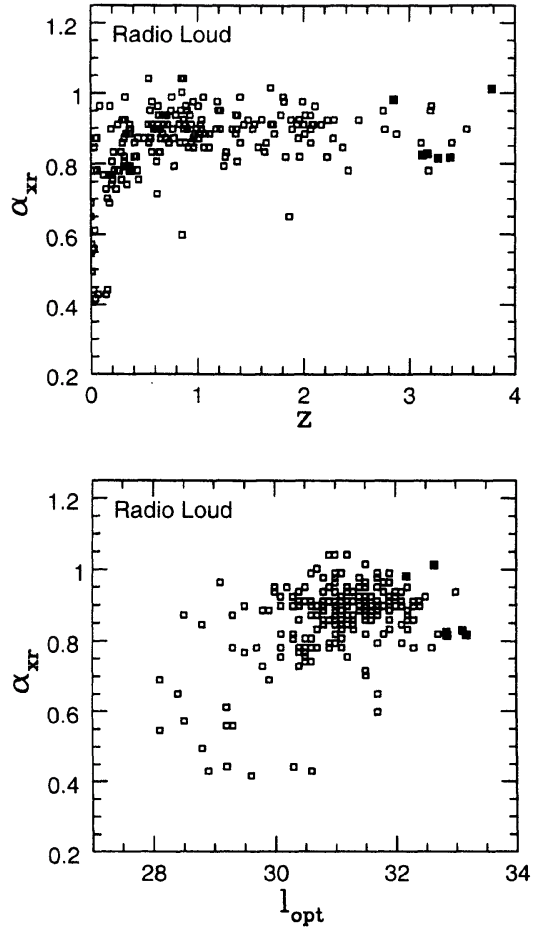

FIG. 15. $\alpha_{\mathrm{xr}}$ as a function of $z$ and $l_{\mathrm{opt}}\left(\mathrm{erg} \mathrm{s}^{-1} \mathrm{~Hz}^{-1}\right)$ for radio loud objects. Symbols are the same as Fig. 13.

which is not as strong in the radio loud objects. For the radio quiet objects, the Kendall Tau and Spearman Rank Correlation tests indicate a correlation with a probability of only $89 \%$ and $80 \%$, respectively. However, again the sample can be divided into two, with significantly different means. For $\log l_{\text {opt }}>32.0$, the mean $\left\langle\alpha_{\text {ox }}\right\rangle=1.67 \pm 0.01$, whereas the lower luminosity objects have $\left\langle\alpha_{\text {ox }}\right\rangle=1.38 \pm 0.05$. This probability that these means are the same is $2.3 \times 10^{-4}$.

To further investigate the relation between $x$-ray and radio emission, we show in Fig. 15 the quantity

$$
\alpha_{\mathrm{xr}}=-\log \left(l_{\mathrm{x}} / l_{\mathrm{r}}\right) / \log \left(\nu_{\mathrm{x}} / \nu_{\mathrm{r}}\right)
$$

as a function of $z$ and $l_{\text {opt }}$ for the radio loud objects only. There is no obvious trend with $z$ or $l_{\text {opt }}$.

The inclusion of the information given by upper limits is clearly important in this discussion. However, for our high redshift sample, essentially all the objects were detected. The statement that the optically selected radio-quiet quasars are relatively $\mathrm{x}$-ray quiet is significant since obviously there is no selection against ones with higher x-ray flux. In Fig. 13, the detected objects should define the lower envelope of the $\alpha_{\text {ox }}-z$ relation, and clearly the high redshift x-ray loud objects are missing. However, there could be objects which are as X-ray bright but optically faint, say $m \sim 21$, and are sufficiently rare not to have been found in deep $\mathrm{x}$-ray surveys to date.

Finally, we note that these conclusions are probably not the result of changes in the mean $x$-ray spectrum with redshift. First, the $\mathrm{x}$-ray colors discussed in Sec. 2.6 are consistent with the spectral index $\alpha_{E} \sim 1$. Second, even if all the 
high redshift objects have large intrinsic absorbing columns (cf. Paper I), the effect on $\alpha_{\text {ox }}$ is small. However, the x-ray luminosity is best determined with the PSPC at an observed energy of $1 \mathrm{keV}$, which for a $z=3$ quasar corresponds to 4 $\mathrm{keV}$; an assumed spectral index is then used to calculate the luminosity at $2 \mathrm{keV}$ in order to derive $\alpha_{\mathrm{ox}}$. As an example, for $1946+768$, we find that for $\alpha_{E}=4$, the value of $\alpha_{\text {ox }}$ decreases from the $\sim 1.9$ to 1.55 ; for $\alpha_{E}=5, \alpha_{\text {ox }}=1.32$. Thus, the x-ray spectral index would have to be somewhat steeper than the observed spectrum of any low redshift quasar to have a strong effect on $\alpha_{\text {ox }}$. Such a steep index would be inconsistent with the hardness ratios discussed above. However, until better x-ray spectra can be obtained for high redshift quasars, this possibility cannot be ruled out.

\section{ACCRETION DISK MODELS}

One of the aims of obtaining multiwavelength spectral energy distributions of quasars is to constrain physical models for the central engine, and and its environs. We defer a detailed discussion of such modeling to a subsequent paper. Instead, in this section, we use the data for two of the objects to illustrate the sort of constraints which may be placed for one particular physical model, the standard, geometrically thin, optically thick accretion disk around a supermassive Kerr black hole. A comparison of this model with Jther physical models (e.g., the free-free model for the blue bump emission, Barvainis 1993) will be discussed in Kuhn et al. (1994) and Kuhn (1994).

Fits were made by eye to the continuum, matching both slope and normalization. The two objects we choose to fit are the two most luminous objects in the sample in the rest UV, $0000-263$ and $0014+813$, since it is the very luminous objects at high redshift which cause the most problems for the black hole paradigm (cf. Turner 1991). 0000-263 is radio quiet and of great interest since it has $z>4 ; 0014+813$ is radio loud, and has a well observed spectral energy distribution.

We assume that the disk radiates locally as a blackbody and that the flux generated in an annulus at a distance $R$ from the center is described by:

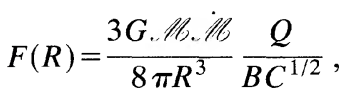

where, $\mathscr{H}$ is the mass of a black hole, $\mathscr{H}$ is the accretion rate, and $Q, B, C$ are the general relativistic correction factors, defined in Novikov \& Thorne (1973) and Page \& Thorne (1974). The generated flux defines the local effective temperature of the blackbody and the local Planck function. General relativity effects on the propagation of light in the vicinity of a black hole were included as a transfer function (Cunningham 1975). The final disk spectrum is an integral over radius of the product of the local emitted flux and the transfer function.

Note that the spectrum at short wavelengths is independent of the disk viscosity when local blackbody emission is àssumed. The defining parameters of the model are the mass of the central black hole, the accretion rate and the inclination angle between the normal to the disk's surface and the
TABLE 11. Summary of accretion disk models.

\begin{tabular}{|c|c|c|c|c|c|c|c|c|c|}
\hline Quasar & $z_{e m}$ & $\mathrm{q}_{o}$ & $\mathrm{H}_{o}{ }^{a}$ & $\mathrm{SED}^{b}$ & $\mathrm{~L} / \mathrm{L}_{E d d^{c}}$ & $\begin{array}{c}\mathrm{L}^{d} \\
\operatorname{ergs~s}^{-1}\end{array}$ & $\begin{array}{c}\mathrm{M}_{B H^{e}} \\
\mathrm{M}_{0}\end{array}$ & $\dot{M}_{\ominus}^{\dot{M}^{f}} \mathrm{yr}^{-1}$ & Comments \\
\hline $0000-26$ & 4.11 & 0 & 50 & $\begin{array}{l}\mathrm{b} \\
\mathrm{c} \\
\mathrm{b} \\
\mathrm{c} \\
\mathrm{b} \\
\mathrm{c}\end{array}$ & $\begin{array}{l}1.0 \text { (Fixed) } \\
1.0 \text { (Fixed) } \\
0.75 \text { (Fixed) } \\
0.75 \text { (Fixed) } \\
0.50 \text { (Fixed) } \\
0.50 \text { (Fixed) }\end{array}$ & $\begin{array}{c}4.6 \times 10^{48} \\
\text { none } \\
5.5 \times 10^{48} \\
\text { none } \\
\text { none } \\
\text { none }\end{array}$ & $\begin{array}{l}3.2 \times 10^{10} \\
5.1 \times 10^{10}\end{array}$ & 250 & $\begin{array}{l}\text { Model too blue } \\
\text { Best fit }\end{array}$ \\
\hline & & $1 / 2$ & 100 & $\begin{array}{l}\mathrm{b} \\
\mathrm{c} \\
\mathrm{b} \\
\mathrm{c} \\
\mathrm{b} \\
\mathrm{c}\end{array}$ & $\begin{array}{l}1.0 \text { (Fixed) } \\
1.0 \text { (Fixed) } \\
0.5 \text { (Fixed) } \\
0.5 \text { (Fixed) } \\
0.1 \text { (Fixed) } \\
0.1 \text { (Fixed) }\end{array}$ & $\begin{array}{l}\sim 10^{47} \\
\sim 10^{48} \\
\sim 10^{47} \\
\sim 10^{48} \\
6.6 \times 10^{46} \\
\text { none }\end{array}$ & $\begin{array}{c}7.0 \times 10^{8} \\
7.0 \times 10^{9} \\
1.4 \times 10^{9} \\
1.4 \times 10^{10} \\
4.5 \times 10^{9}\end{array}$ & $\begin{array}{l}5.4 \\
54 \\
5.4 \\
54 \\
3.5\end{array}$ & $\begin{array}{l}\text { Model too blue } \\
\text { Not good fit } \\
\text { Model too blue } \\
\text { Not good fit } \\
\text { Best Fit }\end{array}$ \\
\hline $0014+81$ & 3.38 & $\begin{array}{c}0 \\
1 / 2\end{array}$ & $\begin{array}{c}50 \\
100\end{array}$ & $\begin{array}{l}\mathrm{c} \\
\mathrm{c}\end{array}$ & $\begin{array}{l}1.0(\text { Fixed) } \\
1.0(\text { Fixed })\end{array}$ & $\begin{array}{c}\text { none } \\
2.2 \times 10^{47}\end{array}$ & $1.5 \times 10^{9}$ & 12 & $\begin{array}{l}\text { Model too red } \\
\text { Best Fit }\end{array}$ \\
\hline
\end{tabular}

Notes to TABLE 11

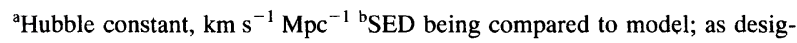
nated in Fig. 8 for $0000-263$, and in Fig. 9 for $0014+813$. ${ }^{c}$ Ratio of total accretion disk luminosity to Eddington luminosity. ${ }^{\mathrm{d}}$ Total accretion disk luminosity. ${ }^{\mathrm{e}}$ Black hole mass for $L / L_{\mathrm{Edd}}$ and $L$. ${ }^{\mathrm{f}}$ Mass accretion rate for $L / L_{\mathrm{Edd}}$ and $L$.

line of sight. We assume that the disk is face-on (the inclination angle is zero) for our further discussions.

The mass and accretion rate cannot be separated based on the observations, since there are many combinations of these parameters resulting in the same spectral shape. The ratio of accretion rate to mass can be related to the ratio of total to Eddington luminosity (Frank et al. 1992):

$$
\frac{L}{L_{\text {Edd }}} \propto \frac{\eta \cdot \mathscr{H}, c^{2}}{. / A},
$$

where $\eta$ is the efficiency of converting the rest mass energy of the accreted matter into radiation. Assuming that the total luminosity cannot be higher than the Eddington luminosity, we can fit the data for different $L / L_{\text {Edd }}$ ratios and estimate the lowest possible central mass, and derive the best combination of the mass and accretion rate to model the observed optical/UV spectral shape and luminosity. We note that if $L / L_{\mathrm{Edd}} \geqslant 0.3$, then the assumption that the disk is geometrically thin is violated (Laor \& Netzer 1989). Table 11 shows the model parameters used to fit the data, including the black hole mass and mass accretion rate. We discuss each object below.

The choice for the outer radius of the accretion disk affects the shape of the continuum at long wavelengths, which for these models is in the rest UV or observed near-IR. We estimated the outer radius by assuming it is determined by the onset of gravitational instability, that is, it is the radius at which the ratio of self-gravity of the disk to the central gravity is greater than unity. Laor \& Netzer (1989) show that the radius at which self-gravity dominates is

$$
R_{\mathrm{SG}} \sim 2150\left(\frac{\mathscr{H} /}{10^{9} \mathscr{C}_{\odot}}\right)^{-2 / 9}\left(\frac{L}{L_{\mathrm{Edd}}}\right)^{4 / 9} \alpha_{\mathrm{Visc}}^{2 / 9} R_{g},
$$

where $R_{g}=G M / c^{2}$. We assumed the viscosity parameter $\alpha_{\text {Visc }}=0.1$ and integrated the accretion disk spectrum to the radius $R_{\mathrm{SG}}$.

In computing the absolute luminosity of the quasars, we used two sets of cosmological parameters: $q_{0}=0$ and $H_{0}=50$ $\mathrm{km} \mathrm{s}^{-1} \mathrm{Mpc}^{-1}$, and $q_{0}=1 / 2$ and $H_{0}=100 \mathrm{~km} \mathrm{~s}^{-1} \mathrm{Mpc}^{-1}$. 


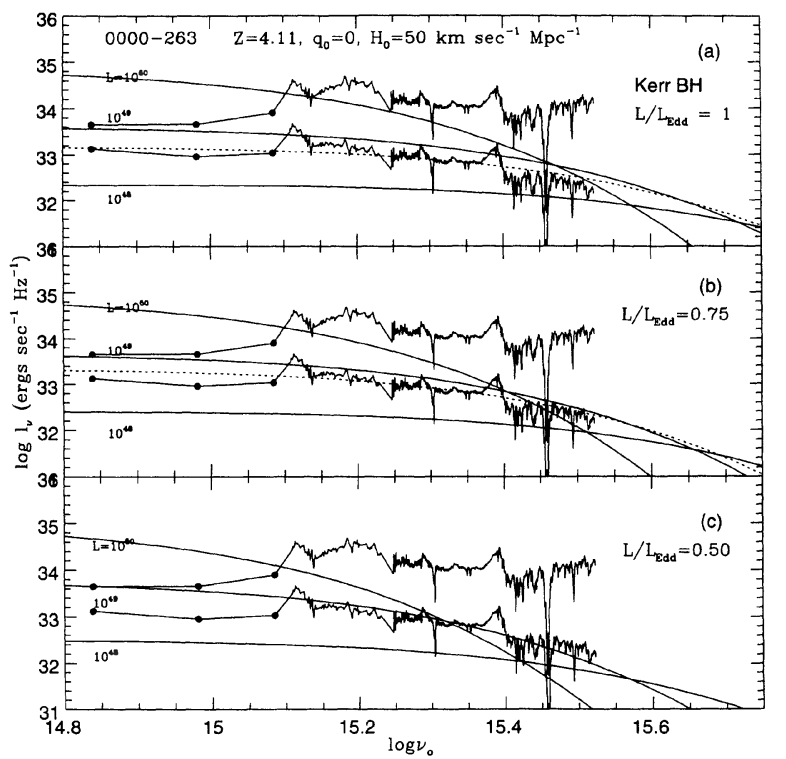

FIG. 16. Kerr black hole and thin accretion disk models compared to the (b) and (c) SED's (of Fig. 8) for 0000-263, assuming $q_{0}=0$ and $H_{0}=50$ $\mathrm{km} \mathrm{s}^{-1} \mathrm{Mpc}^{-1}$. Dashed line shows best "fit": for $L / L_{\mathrm{Edd}}=1, L=4.6 \times 10^{48}$ $\mathrm{erg} \mathrm{s}^{-1}$; for $L / L_{\mathrm{Edd}}=0.75, L=5.5 \times 10^{48} \mathrm{erg} \mathrm{s}^{-1}$.

The first set of cosmological parameters results in the maximum elapsed time since the Big Bang during which quasars can form; the second set results in the minimum plausible luminosity for the objects (Turner 1991).

$$
7.10000-263, z_{e m}=4.11
$$

Figure 16 shows the results for $q_{0}=0$ and $H_{0}=50$ $\mathrm{km} \mathrm{s}^{-1} \mathrm{Mpc}^{-1}$. In Fig. 16(a), the mass accretion rate is fixed at the Eddington value, $L / L_{\text {Edd }}=1$, and the bolometric luminosity of the accretion disk, $L$, is varied. The dereddened spectra $b$ and $c$ are shown. For spectrum c, none of the models give a reasonable fit: they are all too red. For spectrum $b$, the shape is marginally well fit if $L=4.6 \times 10^{48} \mathrm{erg} \mathrm{s}^{-1}$; this is shown as a dotted line in Fig. 16(a). We don't give a formal estimate of the goodness of fit, for reasons which will become clear.

In Fig. 16(b), the analogous plot for $L / L_{\mathrm{Edd}}=0.75$ is shown. Again, spectrum $\mathrm{c}$ is far bluer than any model with any $L$, but spectrum $\mathrm{b}$ is quite well fit if $L=5.5 \times 10^{48}$ $\operatorname{erg~s}^{-1}$, with the shape somewhat better reproduced than the best fit $L / L_{\text {Edd }}=1$ model. In Fig. $16(\mathrm{c})$, the $L / L_{\text {Edd }}=0.5$ models are shown. Here, all the models are redder than the data, for both spectrum $b$ and spectrum $c$. In summary, for this set of cosmological parameters, no model fits spectrum $c$, but spectrum $\mathrm{b}$ is quite well fit with $L / L_{\mathrm{Edd}}=0.75$ and $L \approx 6 \times 10^{48} \mathrm{erg} \mathrm{s}^{-1}$.

Figure 17 shows the same exercise carried out for the other set of cosmological parameters, $q_{0}=1 / 2$ and $H_{0}=100$ $\mathrm{km} \mathrm{s}^{-1} \mathrm{Mpc}^{-1}$. Here spectrum $\mathrm{c}$ can be fit with a model having $L / L_{\mathrm{Edd}}=1$ and $L=10^{49} \mathrm{erg} \mathrm{s}^{-1}$. Spectrum b appears to require a somewhat lower accretion rate, $L / L_{\mathrm{Edd}}=0.1$ and $L=6.6 \times 10^{46} \mathrm{erg} \mathrm{s}^{-1}$ for example, and higher accretion rates appear to result in models that are too blue.

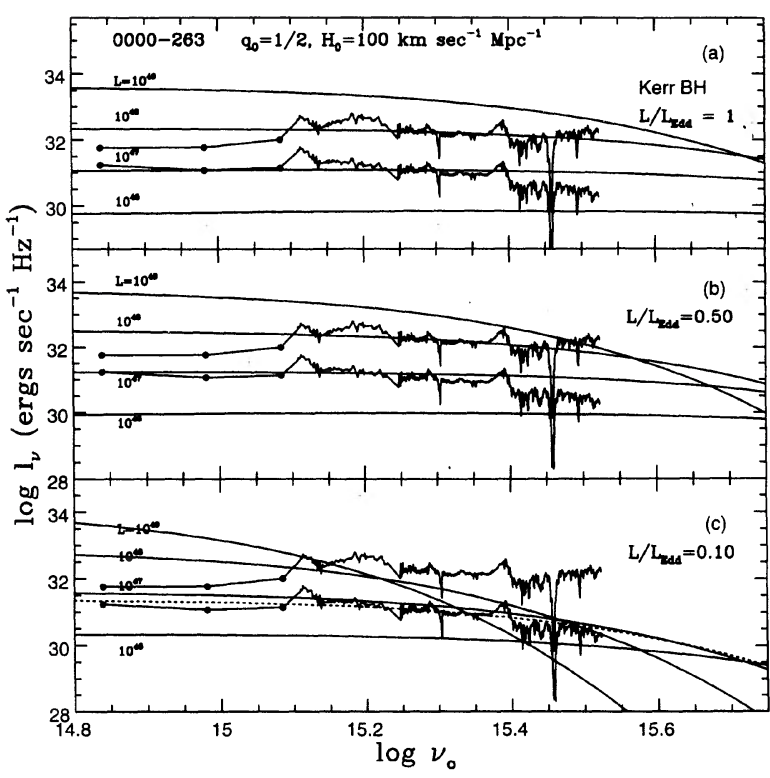

FIG. 17. Same as Fig. 16, except $q_{0}=1 / 2$ and $H_{0}=100 \mathrm{~km} \mathrm{~s}^{-1} \mathrm{Mpc}^{-1}$. Dashed line for $L / L_{\mathrm{Edd}}=0.1$ has $L=6.6 \times 10^{46} \mathrm{erg} \mathrm{s}^{-1}$.

$$
7.20014+813, z_{\text {em }}=3.38
$$

Figure 18 shows the results of fits to the extinction corrected, absorption corrected spectrum "c" of Fig. 9. For $q_{0}=0$ and $H_{0}=50 \mathrm{~km} \mathrm{~s}^{-1} \mathrm{Mpc}^{-1}$, no model fits, whereas for $q_{0}=1 / 2$ and $H_{0}=100 \mathrm{~km} \mathrm{~s}^{-1} \mathrm{Mpc}^{-1}$, a reasonably good fit is given for $L / L_{\mathrm{Edd}}=1$ and $L=2.2 \times 10^{47} \mathrm{erg} \mathrm{s}^{-1}$. As noted above $L / L_{\mathrm{Edd}}=1$ is inconsistent with the assumption that the disk is geometrically thin. However, all $L / L_{\text {Edd }} \leqslant 0.3$ models

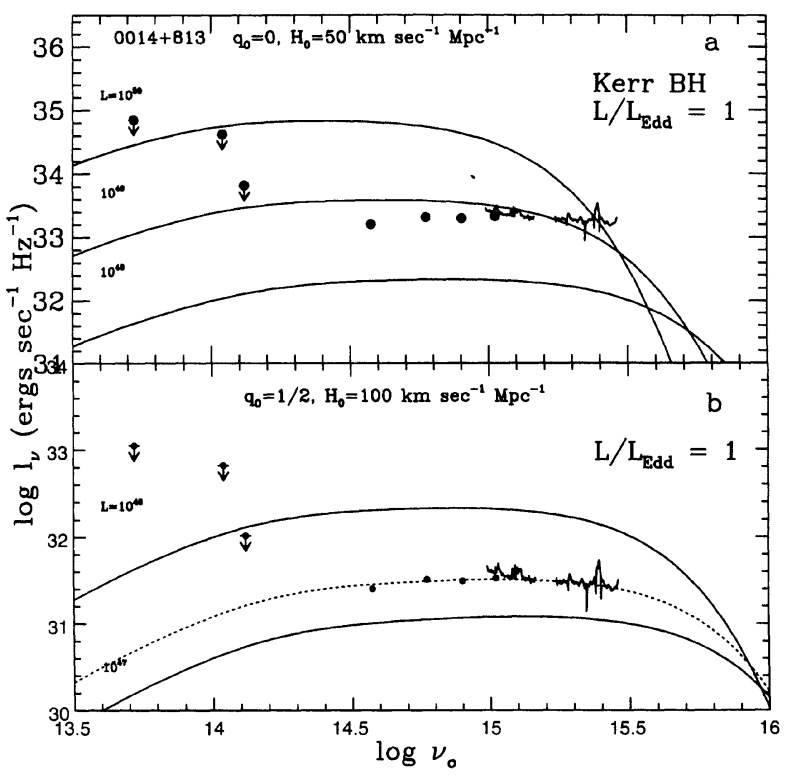

FIG. 18. Kerr black hole and thin accretion disk models compared to the (b) and (c) SED's of Fig. 9 for $0014+813$. Dashed line indicates $L=2.2 \times 10^{47}$ $\mathrm{erg} \mathrm{s}^{-1}$. (a) $q_{0}=0$ and $H_{0}=50 \mathrm{~km} \mathrm{~s}^{-1} \mathrm{Mpc}^{-1}$. (b) $q_{0}=1 / 2$ and $H_{0}=100$ $\mathrm{km} \mathrm{s}^{-1} \mathrm{Mpc}^{-1}$. 
are inconsistent with the data, since they do not extend far enough into the UV. Thus, modifications to the simple face-on Kerr model are required.

\subsection{Results of Disk Fitting}

Table 11 summarizes the results of modeling the data and lists the model parameters, $\mathscr{H}_{\mathrm{BH}}$ and. $\mathscr{H}$, used for the two quasars. The central black hole masses and accretion rates required by observations depend on the assumed cosmology. For $q_{0}=1 / 2$ and $H_{0}=100 \mathrm{~km} \mathrm{~s}^{-1} \mathrm{Mpc}^{-1}$ luminosities are lower, and consequently, the masses and accretion rates of the model are lower. In general, the parameters from our fit agree with the parameters derived solely from total luminosity requirements (Turner 1991). The estimated luminosities are close to the Eddington value, which is expected for the most luminous quasars in the Universe. Again we note that formally the $L / L_{\mathrm{Edd}} \approx 1$ models are inconsistent with the assumption that the disk is geometrically thin.

A surprise is that for both quasars the $J H K$ data, i.e., the optical rest frame, fits quite well, whereas at low $z$ the disks need some extra emission, power-law or dust, to produce sufficient long wavelength emission (Malkan \& Sargent 1982; Sun \& Malkan 1989; Fiore et al. 1994). This could be a significant difference between high and low redshift quasars. We note that in the "underlying power-law" picture (Fiore et al. 1994) the steeper $\alpha_{\text {ox }}$ of the high redshift radio quiet quasars would suggest a lower power-law component in the optical and infrared too, allowing a pure disk component to emerge. This conclusion is true if $R_{\mathrm{SG}}>100 R_{g}$ [Eq. (9)].

We tested only a simple accretion disk model around the Kerr black hole and we considered nnly the face-on case. Changes in the inclination angle will cause a shift of the spectral peak into higher frequencies and give bluer spectra. Thus, if $q_{0}=0$ and $H_{0}=50 \mathrm{~km} \mathrm{~s}^{-1} \mathrm{Mpc}^{-1}$, the spectrum of 0000-263 corrected for known damped Ly $\alpha$ system (with the gas-to-dust ratio equal to the galactic value) may imply higher inclination angles than we have assumed.

While there always exists a pair of parameters which can fit the optical/UV slope and normalization, none of the models is able to fit the observed x-ray flux. In Fig. 19, we show representative model fits which reproduce the IR/optical/UV continuum well, along with the PSPC spectrum. More sophisticated models (including for example, electron scattering and Comptonization in the disk atmosphere, or irradiation of the disk surface) may give a better fit to the data, especially in the higher energy bands. However, given the uncertainty in the cosmological parameters, and the effects of reddening for these quasars, it does not seem likely that precise conclusions about the disk parameters can be drawn.

\section{CONCLUSIONS}

We have observed 14 high redshift quasars with the $R O$ $S A T$ PSPC, and detected 12 of them. We combined the x-ray data with new near-IR and optical spectrophotometry to study the spectral energy distributions of these quasars. The high redshift radio quiet quasars are relatively $\mathrm{x}$-ray quiet compared to their low redshift counterparts, by an amount

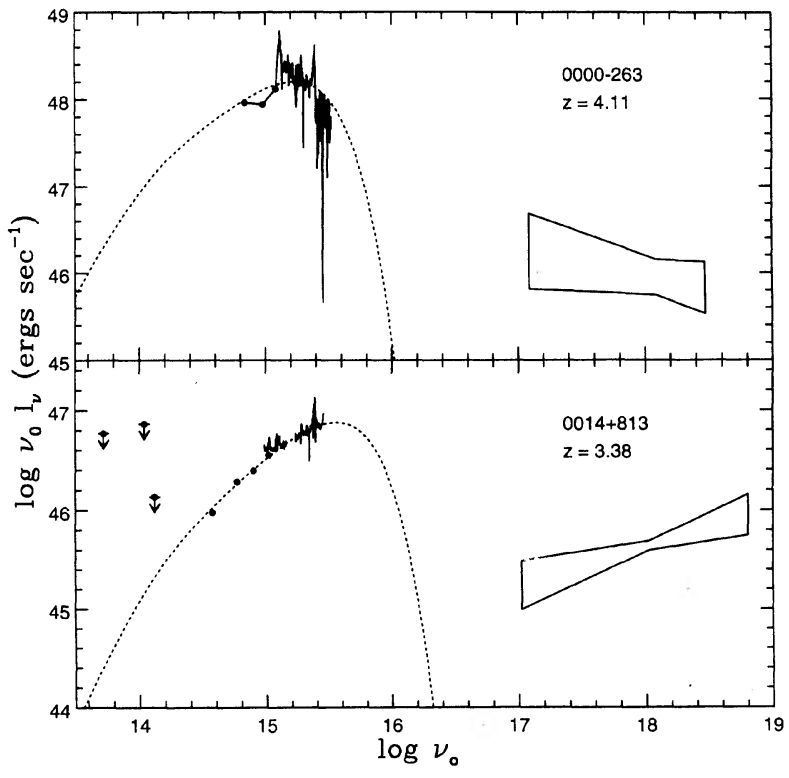

FIG. 19. Representative accretion disk models, compared to $\mathrm{x}$-ray power law fits. For $0000-263$, the dashed line indicates the best fit model from Fig. 16(a). For $0014+813$, the dotted line indicates the best fit model from Fig. 18 (b).

consistent with an extrapolation of the dependence of $\alpha_{\text {ox }}$ on $l_{\text {opt }}$ observed at lower $z$. The radio loud high redshift quasars, on the other hand, are more x-ray loud than expected, given their $l_{\text {opt }}$, if they are comparable to steep spectrum, compact radio sources at low redshift. The $\mathrm{x}$-ray hardness ratios of the objects in our sample suggest that the radio quiet and radio loud quasars at $z \approx 3$ have different $\mathrm{x}$-ray spectra. The radio loud high redshift quasars show significant absorption, which is not seen at low redshift, whereas the radio quiet quasars either have substantial absorption and steeper $\alpha_{E}$ 's or no absorption and similar $\alpha_{E}$ 's than their low redshift counterparts. Aside from these trends, the spectral energy distributions of the high redshift objects are similar to those of low redshift quasars.

Alain Porter suggested we observe $1946+77$. We thank Pat Henry for communicating results before publication. Data was obtained with the Multiple Mirror Telescope, a joint facility of the University of Arizona and the HarvardSmithsonian Astrophysical Observatory. It is a pleasure to thank the staffs of the MMTO and Steward Observatory for their assistance. This research has made use of the NASA/ IPAC Extragalactic Database (NED) which is operated by the Jet Propulsion Laboratory, California Institute of Technology, under contract with the National Aeronautics and Space Administration. This research has also made use of the Einstein On-Line Service, Smithsonian Astrophysical Observatory; the High Energy Astrophysics Science Archive Research Center, Goddard Space Flight Center; the IRAS archive, IPAC; SIMBAD; and the Hubble Space Telescope data archive, Space Telescope Science Institute. IPAC is funded by NASA as part of the Extended Mission Program, under 
contract to JPL. This work was supported by NASA Grants NAGW-2201 (LTSARP), NAG5-1872, NAG5-1536, and NAG5-1680 (ROSAT), and NASA contracts NAS8-39073
(ASC) and NAG5-30934 (RSSDC). This work was also supported by NSF Grants RII-8800660, INT-9010583, and AST9058510, and a gift from Sun Microsystems.

\section{REFERENCES}

Adam, G. 1985, A\&AS, 61, 225

Antonucci, R. R. J., Kinney, A. C., \& Ford, H. C. 1989, ApJ, 342, 64 Atwood, B., Baldwin, J. A., \& Carswell, R. F. 1985, ApJ, 292, 58 Avni, Y., \& Tananbaum, H. 1982, ApJ, 262, L17

Avni, Y., \& Tananbaum, H. 1986, ApJ, 305, 83

Bahcall, J. N., et al. 1992a, ApJ, 392, L1

Bahcall, J. N., et al. 1992b, ApJ, 400, L51

Barvainis, R. 1993, ApJ, 412, 513

Bechtold, J. 1994, ApJ, 91, 1

Bechtold, J., et al., 1994, AJ (in press)

Becker, R. H., Helfand, D. J., \& White, R. L. 1992, AJ, 104, 531

Becker, R. H., White, R. L., \& Edwards, A. L. 1991, ApJS, 75, 1

Bierman, P. L., Schaff, R., Pietsch, W., Schmutzler, T., Witzel, A., \& Kuhr, H. 1992, A\&AS, 96, 339

Bohlin, R. 1978, ApJ, 224, 132

Boyle, B. J. 1993, The Environment and Evolution of Galaxies, edited by $\mathbf{J}$. M. Shull and H. A. Thronson (Kluwer, Dordrecht), p. 433

Boyle, B. J., Griffiths, R. E., Shanks, T., Stewart, G. C., \& Georgantopoulos, I. 1993, MNRAS, 260, 49

Browne, I. W. A., \& Perley, R. A. 1986, MNRAS, 222, 149

Ciliegi, P., et al. 1994 (in preparation)

Condon, J. J., Condon, M. A., \& Hazard, C. 1982, AJ, 87, 739

Condon', J. J., Condon, M. A., Jauncey, D. L., Smith, M. G., Turtle, A. J., \&

Wright, A. E. 1981, ApJ, 244, 5

Condon, J. J., Hicks, P. D., \& Jauncey, D. L. 1977, AJ, 82, 692

Cunningham, C. T. 1975, ApJ, 202, 788

Cutri, R. M., Huchra, J. P., \& Low, F. J. 1994, ApJ, 424, L65

Della Ceca, R., \& Maccacaro T. 1991, ASP Conference Series 21, The Space

Distribution of Quasars, edited by D. Crampton (Astron. Soc. Pacific, San Francisco), p. 150

Elvis, M., Green, R. F., Bechtold, J., Schmidt, M., Neugebauer, G., Soifer,

B. T., Matthews K., \& Fabbiano G. 1986, ApJ, 310, 291

Elvis, M., Fiore, F., Wilkes, B., McDowell, J., \& Bechtold, J. 1994a, ApJ, 422, 60 (Paper I)

Elvis, M., Lockman, F. J., \& Wilkes, B. J. 1989, ApJ, 97, 777

Elvis, M., et al. 1994b, ApJS (in press)

Fink, H. H., \& Briel, U. G. 1993, A \& A, 274, L45

Fiore, F., et al. 1994, ApJ (submitted)

Frank, J., King, A. R. \& Raine, D. J. 1992, Accretion Power in Astrophysics, Second Edition (Cambridge University)

Fugmann, W., \& Meisenheimer, K. 1988, A\&AS, 76, 145

Ge, J., \& Bechtold, J. 1994, in preparation

Ghosh, K. K., \& Soundararajaperumal, S. 1992, A\&A, 265, 413

Green, P. J., Anderson, S. F., \& Ward, M. J. 1991, MNRAS, 254, 30

Gregory, P. C., \& Condon, J. J. 1991, ApJS, 75, 1011

Hagen, H.-J., et al. 1992, A\&A, 253, L5

Hasinger G., Schmidt, M., \& Trumper J. 1991, A\&A, 246, L2

Hazard, C., \& McMahon, R. 1985, Nature, 314, 238

Hazard, C., McMahon, R. G., \& Sargent, W. L. W. 1986, Nature, 322, 38

Hazard, C., Terlevich, R., McMahon, R., Turnshek, D., Foltz, C., Stocke, J.,

\& Weymann, R. 1984, MNRAS, 211, 45P

Heiles, C., \& Cleary M. N. 1979, AuJPS, 47, 1

Henry, J. P., Gioia, I. M., \& Boehringer, H. 1994, AJ, 107, 1270

Hewitt, A., \& Burbidge, G. 1987 ApJS, 63, 1

Hewitt, A., \& Burbidge, G. 1993, ApJS, 87, 451

Hoag, A. A., \& Smith, M. G. 1977, ApJ, 217, 362

Hyland, A. R., \& Allen, D. A. 1982, MNRAS, 199, 943

Impey, C. D., \& Tapia, S. 1990, ApJ, 354, 124

Jauncey, D. L., Wright, A. E., Peterson, B. A., \& Condon, J. J. 1978, ApJ, 223, L1

Kriss, G. A., \& Canizares, C. R. 1985, ApJ, 297, 177

Kollgaard, R., et al. 1994, ApJ (in press)
Kuhn, O. 1994, Ph.D. thesis

Kuhn, O., Bechtold, J., Cutri, R., Elvis, M., \& Rieke, M. 1994, ApJ (in press)

Kuhr, H. 1977, A\&AS, 24, 139

Kuhr, H., Liebert, J. W., Strittmatter, P. A., Schmidt, G. D., \& Mackay, C. 1983, ApJ, 275, L33

Kuhr, H., McAlary, C. W., Rudy, R. J., Strittmatter, P. A., \& Rieke, G. H. 1984, ApJ, 284, L5

Kuhr, H., Stocke, J. T., Strittmatter, P. A., Bartel, N., Eckart, A., Schalinski, C., Witzel, A., \& Biermann, P. 1986, ApJ, 302, 52

Lanzetta, K. M., Turnshek, D. A., \& Sandoval, J. 1993, ApJS, 84, 109

Laor, A., \& Netzer, H. 1989, MNRAS, 238, 897

Lawson, A. J., Turner, M. J. L., William, O. R., Stewart, G. C., \& Saxton, R. D. 1992, MNRAS, 259, 743

Maccacaro, T., Della Ceca, R., Gioia, I. M., Morris, S. L., Stocke, J. T., \& Wolter, A. 1991, ApJ, 374, 117

Maccacaro, T., Gioia, I. M., Wolter, A., Zamorani, G., \& Stocke, J. T. 1988, ApJ, 326, 680

Magain, P., Surdej, J., Vanderriest, C., Prienne, B., \& Hutsemekers, D. 1992, A\&A, 253, L13

Malkan, M. A., \& Sargent, W. L. W. 1982, ApJ, 254, 22

Maslowski, J., Pauliny-Toth, I. I. K., Witzel, A., \& Kuhr, H. 1984, A\&A, 139, 85

McGlynn, T. A., Tennant, A. F., Shafer, R. A., \& Stewart G. C. 1985, SSRv, 40, 633

Morrison, R., \& McCammon, D. 1983, ApJ, 270, 119

Morton, D. C., Savage, A., \& Bolton, J. G. 1978, MNRAS, 185, 735

Netzer, H. 1990, Active Galactic Nuclei, Saas-Fee Advanced Course 20, edited by T. J.-L. Courvoisier and M. Mayor (Springer; New York), p. 57

Neugebauer, G., Green, R. F., Matthews K., Schmidt M., Soifer, B. T., \& Bennett J. 1987, ApJS, 63, 615

Neugebauer, G., Miley, G. K., Soifer, B. T., \& Clegg, P. E. 1986, ApJ, 308, 815

Novikov, I. D., \& Thorne, K., S. 1973, Black Holes, edited by C. De Witt and B. De Witt (Gordon \& Breach, New York)

O’Dea, C. P., Baum, S. A., \& Stanghellini, C. 1991, ApJ, 380, 66

Oke, J. B., \& Korycansky, D. G. 1982, ApJ, 255, 11

Osmer, P. A. 1977, ApJ, 218, L89

Osmer, P. S., \& Smith, M. G. 1976, ApJ, 210, 267

Osmer, P. S., \& Smith, M. G. 1980, ApJS, 42, 333

Page, D. N., \& Thorne, K. S. 1974, ApJ, 191, 499

Patnaik, A. R., Browne, I. W. A., Wilkinson, P. N., \& Wrobel, J. M. 1992, MNRAS, 254, 655

Pauliny-Toth, I. I. K., Witzel, A., Preuss, E., Kuhr, H., Kellerman, K. I., Fomalont, E. B., \& Davis, M. M. 1978, AJ, 83, 451

Pei, Y. C., Fall, S. M., \& Bechtold, J. 1991, ApJ, 378, 6

Pfefferman E., et al. 1987, Proc. SPIE, 733, 519

Peterson, B. A., Savage, A. Jauncey, D. L., \& Wright, A. E. 1982, ApJ, 260, L27

Pettini, M., Smith, L. J., \& Hunstead, R. W. 1994, ApJ, 426, 79

Preston, R. A., Morabito, D. D., Williams, J. G., Faulkner, J., Jauncey, D. L., \& Nicolson, G. D. 1985, AJ, 90, 1599

Preston, R. A., et al. 1989, AJ, 98, 1

Quiniento, Z. M., \& Cerosimo, J. C. 1993, A\&AS, 97, 435

Quiniento, Z. M., Cerosimo, J. C., \& Colomb, F. R. 1988, A\&AS, 76, 21

Rauch, M., et al. 1992, ApJ, 390, 387

Rieke, G. H., Elston, R. J., Lebofsky, M. J., \& Walker, C. E. 1987, Infrared Astronomy with Arrays, edited by C. G. Wynn-Williams and E. E. Becklin (University of Hawaii, Honolulu), 69

Rieke, G. H., \& Lebofsky, M. J. 1985, ApJ, 288, 618

Robson, E. I., Gear, W. K., Smith, M. G., Ade, P. A. R., \& Nolt, I. G. 1985, MNRAS, 213, 355 
Rodriquez-Espinoza, J. M., Stanga, R. M., \& Moorwood, A. F. M. 1988 A\&A, 192, 13

Saikia, D. J., \& Shastri, P. 1984, MNRAS, 211, 47

Sanders, D. B., et al. 1989, ApJ, 347, 29

Sanduleak, N., \& Pesch, P. 1989, PASP, 101, 1081

Sargent, W. L. W., Filippenko, A. V., Steidel, C., Hazard, C., \& McMahon, R. G. 1986, Nature, 322, 40

Sargent, W. L. W., Steidel, C. C., \& Boksenberg, A. 1989, ApJS, 69, 703

Saucedo, J., \& Bechtold, J. 1994, ApJ (submitted)

Savage, B. D., \& Mathis, J. S. 1979, ARA\&A, 17, 73

Savage, A., Jauncey, D. L., White, G. L., Peterson, B. A., Peters, W. L.,

Gulkis, S., \& Condon, J. J. 1990, AuJPh, 43, 241

Savage, B. D., et al. 1993, ApJ, 413, 116

Schneider, D. P., Schmidt, M., \& Gunn, J. E. 1989, AJ, 98, 1507

Schneider, D. P., et al. 1992a, PASP, 104, 678

Schneider, D. P., Van Gorkom, J. H., Schmidt, M., \& Gunn, J. E. 1992b, AJ, 103,1451

Shanks, T., Georgantopoulos, I., Steward, G. C., Pounds, K. A., Boyle, B. J., \& Griffiths, R. E. 1991, Nature, 353, 315

Smith, A. 1984, EXOSAT Express No. 5, p. 48

Soifer, B. T., Neugebauer, G., Oke, J. B., Matthews, K., \& Lacy, J. H. 1983, ApJ, 265, 18

Stark, A. A., Gammie, C. F., Wilson, R. W., Bally, J., Linke, R., Heiles, C., \& Hurwitz, M. 1989, ApJS, 79, 77

Steidel, C. C. 1990 , ApJS, 72,1

Steidel, C. C., \& Hamilton, D. 1992, AJ, 104, 941

Steppe, H., Salter, C. J., Chini, R., Kraysa, E., Brunswig, W., \& Lobato Perez, J. 1988, A\&AS, 75, 317
Sun, W-H., \& Malkan, M. 1989, ApJ, 346, 68

Trumper, J. 1983, Adv. Space Res., 2, 241

Turner, E. L. 1991, AJ, 101, 5

Turnshek, D. A., Macchetto, F., Bencke, M. V., Hazard, C., Sparks, W. B., \& McMahon, R. G. 1991, ApJ, 382, 26

Veron-Cetty, M.-P., \& Veron, P. 1989, ESO Scientific Report, No. 7

Visnovsky, K. L., Impey, C. D., Foltz, C. B., Hewett, P. C., Weymann, R. J., \& Morris, S. L. 1992, ApJ, 391, 560

Wall, J. V., \& Peacock, J. A. 1985, MNRAS, 216, 173

Webb, J. K., Barcons, X., Carswell, R. F., \& Parnell, H. C. 1992, MNRAS, 255,319

Webb, J. K., Parnell, H. C., Carswell, R. F., McMahon, R. G., Irwin, M. H., Hazard, C., Ferlet, R., \& Vidal-Madjar, A. 1988, Messenger, 51, 15

Wehrle, A. E., Morabito, D. D., \& Preston, R. A. 1984, AJ, 89, 336

Whelan, J. A. J., Smith, M. G., \& Carswell, R. F. 1979, MNRAS, 189, 363

White, R. L., \& Becker, R. H. 1992, ApJS, 79, 331

Wilkes, B. J., Elvis, M., Fiore, F., McDowell, J. C., Tananbaum, H., \& Lawrence, A. 1992, ApJ, 393, L1

Wilkes, B. J., Elvis, M., \& McHardy I. 1987, ApJ, 321, L23

Wilkes, B. J., Tananbaum, H., Avni, Y., Worrall, D. M., Oey, M. S., \& Flanagan, J. 1994, ApJS (in press)

Wills, B. J., Wills, D., Breger, M. Antonucci, R. R. J., \& Barvainis, R. 1992, ApJ, 398, 454

Worrall, D. M., Giommi, P., Tananbaum, H., \& Zamorani, G. 1987, ApJ, 313,596

Wright, A. E., Wark, R. M., Troup, E., Otrupcek, R., Jenning, D., Hunt, A., \& Cooke, D. J. 1991, MNRAS, 251, 330

Zamorani, G., et al. 1981, ApJ, 245, 357 